\title{
Multicarrier Block-Spread CDMA for Broadband Cellular Downlink
}

\author{
Frederik Petré \\ Wireless Research, Interuniversity MicroElectronics Center (IMEC), Kapeldreef 75, 3001 Leuven, Belgium \\ Email: frederik.petre@imec.be
}

\section{Geert Leus}

Electrical Engineering, Mathematics and Computer Science, Delft University of Technology (TUDelft), Mekelweg 4, 2628 CD Delft, The Netherlands

Email:leus@cas.et.tudelft.nl

\author{
Marc Moonen \\ Department of Electrical Engineering (ESAT), Katholieke Universiteit Leuven (KULeuven), Kasteelpark Arenberg 10, \\ 3001 Leuven, Belgium \\ Email:marc.moonen@esat.kuleuven.ac.be
}

\section{Hugo De Man}

Interuniversity MicroElectronics Center (IMEC), Kapeldreef 75, 3001 Leuven, Belgium

Email:hugo.deman@imec.be

\section{Received 6 March 2003; Revised 7 November 2003}

\begin{abstract}
Effective suppression of multiuser interference (MUI) and mitigation of frequency-selective fading effects within the complexity constraints of the mobile constitute major challenges for broadband cellular downlink transceiver design. Existing wideband direct-sequence (DS) code division multiple access (CDMA) transceivers suppress MUI statistically by restoring the orthogonality among users at the receiver. However, they call for receive diversity and multichannel equalization to improve the fading effects caused by deep channel fades. Relying on redundant block spreading and linear precoding, we design a so-called multicarrier block-spread- (MCBS-)CDMA transceiver that preserves the orthogonality among users and guarantees symbol detection, regardless of the underlying frequency-selective fading channels. These properties allow for deterministic MUI elimination through low-complexity block despreading and enable full diversity gains, irrespective of the system load. Different options to perform equalization and decoding, either jointly or separately, strike the trade-off between performance and complexity. To improve the performance over multi-input multi-output (MIMO) multipath fading channels, our MCBS-CDMA transceiver combines well with space-time block-coding (STBC) techniques, to exploit both multiantenna and multipath diversity gains, irrespective of the system load. Simulation results demonstrate the superior performance of MCBS-CDMA compared to competing alternatives.
\end{abstract}

Keywords and phrases: multicarrier CDMA, broadband cellular system, frequency-selective fading channels, equalization, MIMO, space-time block coding.

\section{INTRODUCTION}

The main drivers toward future broadband cellular systems, like high-speed wireless internet access and mobile multimedia, require much higher data rates in the downlink (from base to mobile station) than in the uplink (from mobile to base station) direction. Given the asymmetric nature of most of these broadband services, the capacity and performance bottlenecks clearly reside in the downlink of these future systems. Broadband cellular downlink communications poses three main challenges to successful transceiver design. First, for increasing data rates, the underlying multipath channels become more time dispersive, causing intersymbol interference (ISI) and interchip interference (ICI), or, equivalently, frequency-selective fading. Second, due to the increasing success of future broadband services, more users will try to access the common network resources, causing multiuser interference (MUI). Both ISI/ICI and MUI are important performance limiting factors for future broadband cellular systems, because they determine their capabilities in dealing with high data rates and system loads, respectively. Third, cost, size, and power consumption issues put severe constraints on the receiver complexity at the mobile station (MS). 
Direct-sequence (DS) code division multiple access (CDMA) has emerged as the predominant air interface technology for the $3 \mathrm{G}$ cellular standard [1], because it increases capacity and facilitates network planning in a cellular system, compared to conventional multiple access techniques like frequency-division multiple access (FDMA) and timedivision multiple access (TDMA) [2]. In the downlink, DSCDMA relies on the orthogonality of the spreading codes to separate the different user signals. However, ICI destroys the orthogonality among users, giving rise to MUI. Since the MUI is essentially caused by the multipath channel, linear chip-level equalization, followed by correlation with the desired user's spreading code, allows to suppress the MUI $[3,4,5,6]$. However, chip equalizer receivers suppress MUI only statistically, and require receive diversity to cope with the effects caused by deep channel fades [7, 8].

On the other hand, it is well known that orthogonal frequency-division multiplexing (OFDM), also called multicarrier (MC) modulation, with cyclic prefixing (CP) constitutes an elegant solution to combat the wireless channel impairments $[9,10,11]$. It converts a frequency-selective channel into a number of parallel flat fading channels by multiplexing blocks of information symbols on orthogonal subcarriers using implementation efficient fast Fourier transform (FFT) operations. Hence, the complex equalizer commonly encountered in single-carrier (SC) systems reduces to a set of parallel and independent single-tap equalizers. However, OFDM, in itself, does not extract frequency diversity, but calls for bandwidth overconsuming forward error correction (FEC) coding techniques to enable frequency diversity [12]. Furthermore, OFDM as such does not support multiple users but requires a multiple access technique on top of it.

In this paper, we propose a novel MC-CDMA transceiver that synergistically combines the advantages of DS-CDMA and OFDM to tackle the challenges of broadband cellular downlink communications. By capitalizing on the general concepts of redundant block spreading and linear precoding, our so-called multicarrier block-spread- (MCBS-)CDMA transceiver possesses three unique properties compared to competing alternatives (Section 2). First, by $\mathrm{CP}$ or zero padding (ZP) the block-spread symbol blocks, our MCBSCDMA transceiver preserves the orthogonality among users, regardless of the underlying time-dispersive multipath channels. This property allows for deterministic (as opposed to statistical) MUI elimination through low-complexity and channel-independent block despreading. Second, redundant linear precoding guarantees symbol detectability and full frequency-diversity gains, thus robustifying the transmission against deep channel fades. Assuming perfect channel state information (CSI) at the receiver, different equalization and decoding options, ranging from linear over decisiondirected to maximum likelihood (ML) detection, strike the trade-off between performance and complexity (Section 3). Finally, our transceiver exhibits a rewarding synergy with multiantenna techniques, to increase the spectral efficiency and to improve the link reliability of multiple users in a broadband cellular network (Section 4). Simulation results demonstrate the outstanding performance of the proposed transceiver compared to competing alternatives (Section 5).

Several other MC-CDMA techniques that also combine CDMA with OFDM have recently gained increased momentum as candidate air interface for future broadband cellular systems [13]. Three different flavours of MC-CDMA exist, depending on the exact position of the CDMA and the OFDM component in the transmission scheme. The first variant, called MC-CDMA, performs the spreading operation before the symbol blocking (or serial-to-parallel conversion), which results in a spreading of the information symbols across the different subcarriers [14, 15, 16]. However, like classical DS-CDMA, MC-CDMA does not enable full frequency-diversity gains. The second variant, called MCDS-CDMA, executes the spreading operation after the symbol blocking, resulting in a spreading of the information symbols along the time axis of the different subcarriers [17, 18]. However, like classical OFDM, MC-DS-CDMA necessitates bandwidth overconsuming FEC coding plus frequencydomain (FD) interleaving to mitigate frequency-selective fading. The third variant, called multitone (MT) DS-CDMA, performs the spreading after the OFDM modulation such that the resulting spectrum of each subcarrier no longer satisfies the orthogonality condition [19]. Hence, MT-DSCDMA suffers from ISI and intertone interference (ITI), as well as MUI, and requires expensive multiuser detection techniques to achieve a reasonable performance. Finally, alternative MUI-free MC transceivers, like AMOUR [20] and generalized multicarrier (GMC) CDMA [11], rely on an orthogonal frequency-division multiple access- (OFDMA-)like approach to retain the orthogonality among users, regardless of the underlying multipath channels. Unlike our MCBSCDMA transceiver, these transceivers do not inherit the nice properties of CDMA related to universal frequency reuse ${ }^{1}$ in a cellular network, such as increased capacity and simplified network planning.

\section{Notation}

We use roman letters to represent scalars, lower boldface letters to denote column vectors (i.e., blocks), and upper boldface letters to denote matrices (i.e., a collection of blocks). $(\cdot)^{*},(\cdot)^{T}$, and $(\cdot)^{H}$ represent conjugate, transpose, and Hermitian, respectively. Further, $|\cdot|$ and $\|\cdot\|$ represent the absolute value and Frobenius norm, respectively. We reserve E $\{\cdot\}$ for expectation and $\lfloor\cdot\rfloor$ for integer flooring. Subscripts $n_{t}$ and $n_{r}$ point to the $n_{t}$ th transmit and the $n_{r}$ th receive antenna, respectively. Superscript $m$ points to the $m$ th user. Argument $i$ denotes symbol index for symbol scalar sequences and symbol block index for symbol block sequences. Likewise, argument $n$ denotes chip index for chip scalar sequences and chip block index for chip block sequences. Tilded letters $\tilde{x}$ denote FD signals and upperlined letters $\bar{x}$ denote space-time block-encoded signals at the transmitter and block-despread

\footnotetext{
${ }^{1}$ Universal frequency reuse, also called frequency reuse of one-in-one, is a unique attribute of CDMA systems, which refers to the reuse of the same frequencies in neighbouring cells.
} 


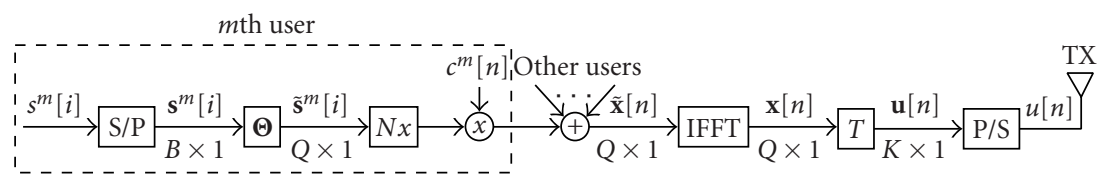

FIgURE 1: MCBS-CDMA downlink transmission scheme.

signals at the receiver. Acuted letters $x$ denote space-time block-decoded signals at the receiver. Hatted letters $\hat{x}$ denote soft estimates, whereas hatted and underlined letters $\underline{\hat{x}}$ denote hard estimates.

\section{MCBS-CDMA TRANSCEIVER DESIGN}

Effective suppression of MUI and mitigation of ISI and frequency-selective fading, within the complexity constraints of the MS, pose major challenges to transceiver design for the broadband cellular downlink application. To tackle these challenges, we propose a novel MC-CDMA transceiver that combines two specific CDMA and OFDM concepts, namely, block-spread CDMA and linearly-precoded OFDM. The resulting so-called MCBS-CDMA transceiver exhibits two unique properties compared to competing alternatives. First, by relying on block-spread CDMA, MCBS-CDMA preserves the orthogonality among users, even after propagation through a time-dispersive multipath channel. This property allows for deterministic (as opposed to statistical) MUI elimination at the receiver through low-complexity block despreading. Second, by relying on linearly-precoded OFDM, MCBS-CDMA mitigates ISI and guarantees symbol detection, regardless of the underlying frequency-selective multipath channel. This property enables full frequency-diversity gains and, hence, robustness against frequency-selective fading at the receiver, through ML single-user equalization. Furthermore, different single-user equalization options, ranging from linear over decision-directed to ML detection, strike the trade-off between performance and complexity.

This section is organized as follows. Section 2.1 introduces the MCBS-CDMA downlink transmission scheme, and motivates the different operations involved. Section 2.2 demonstrates how our MCBS-CDMA transceiver enables MUI-resilient reception over frequency-selective multipath channels. Finally, Section 2.3 argues the need for single-user equalization and guaranteed symbol detection.

\subsection{MCBS-CDMA downlink transmission}

We consider a single cell of a cellular system with a base station (BS) serving $M$ active MSs within its coverage area. For now, we limit ourselves to the single-antenna case and defer the multiantenna case to Section 4 . The block diagram in Figure 1 describes the MCBS-CDMA downlink transmission scheme (where only the $m$ th user is explicitly shown) that transforms the $M$ user data symbol sequences $\left\{s^{m}[i]\right\}_{m=1}^{M}$, with a rate $1 / T_{s}$, into the multiuser chip sequence $u[n]$, with a rate $1 / T_{c}$. Apart from the user multiplexing and the IFFT, the MCBS-CDMA transmission scheme performs three ma- jor operations, namely, linear precoding, block spreading, and adding transmit redundancy. Since our scheme belongs to the general class of block transmission schemes, the $m$ th user's data symbol sequence $s^{m}[i]$ is first serial-to-parallel converted into blocks of $B$ symbols, leading to the symbol block sequence $\mathbf{s}^{m}[i]:=\left[s^{m}[i B], \ldots, s^{m}[(i+1) B-1]\right]^{T}$.

The first operation involves complex-field linear precoding, where the encoding is performed over the complex field rather than over the Galois field, as done traditionally $[21,22]$. Unlike MC-CDMA that spreads the information symbols across the subcarriers employing a user-specific spreading code $[14,15,16]$, MCBS-CDMA precodes the information symbols on the different subcarriers employing a linear precoding matrix. Specifically, the information blocks $\mathbf{s}^{m}[i]$ are linearly precoded by a $Q \times B$ matrix $\Theta$ to yield the $Q \times 1$ precoded symbol blocks:

$$
\tilde{\mathbf{s}}^{m}[i]:=\boldsymbol{\Theta} \cdot \mathbf{s}^{m}[i]
$$

where $Q$ is the number of subcarriers, and $\Theta$ is a para-unitary matrix, that is, $\boldsymbol{\Theta}^{H} \cdot \boldsymbol{\Theta}=\mathbf{I}_{B}$. The linear precoding can be either redundant $(Q>B)$ or nonredundant $(Q=B)$. For conciseness, we limit our discussion to redundant precoding, but the proposed concepts apply equally well to nonredundant precoding. As we will show later, linear precoding guarantees symbol detection and maximum frequencydiversity gains, and thus robustifies the transmission against frequency-selective fading.

The second operation entails a block-spreading operation, which is also depicted in Figure 1. Unlike DS-CDMA and MC-CDMA that rely on classical symbol spreading (operating on a scalar symbol), MCBS-CDMA relies on block spreading (operating on a block of symbols). Specifically, the block sequence $\widetilde{\mathbf{s}}^{m}[i]$ is block spread by a factor $N$ with the user composite code sequence $c^{m}[n]$, which is the multiplication of a short (periodic) orthogonal Walsh-Hadamard spreading code that is MS specific and a long (aperiodic) overlay scrambling code that is BS specific. The chip block sequences of the different active users are added, resulting in the multiuser chip block sequence:

$$
\tilde{\mathbf{x}}[n]=\sum_{m=1}^{M} \tilde{\mathbf{s}}^{m}[i] c^{m}[n],
$$

where the symbol block index $i$ relates to the chip block index $n$ through $i=\lfloor n / N\rfloor$. The block spreading operation is also illustrated in Figure 1, where the $N \times$ replicator repeats the symbol block at its input $N$ times. Collecting $N$ consecutive chip blocks, $\tilde{\mathbf{x}}[n]$, into $\tilde{\mathbf{X}}[i]:=[\tilde{\mathbf{x}}[i N], \ldots, \tilde{\mathbf{x}}[(i+1) N-1]]$, 


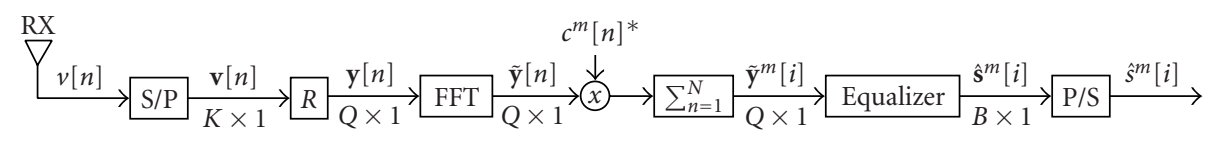

FIgURE 2: MUI-resilient MCBS-CDMA downlink reception scheme.

we obtain the symbol block level equivalent of (2), that is:

$$
\tilde{\mathbf{X}}[i]=\sum_{m=1}^{M} \tilde{\mathbf{s}}^{m}[i] \cdot \mathbf{c}^{m}[i]^{T}=\tilde{\mathbf{S}}[i] \cdot \mathbf{C}[i]^{T},
$$

where $\mathbf{c}^{m}[i]:=\left[c^{m}[i N], \ldots, c^{m}[(i+1) N-1]\right]^{T}$ is the $m$ th user's composite code vector used to block-spread its data symbol block $\tilde{\mathbf{s}}^{m}[i], \tilde{\mathbf{S}}[i]:=\left[\tilde{\mathbf{s}}^{1}[i], \ldots, \tilde{\mathbf{s}}^{M}[i]\right]$ collects the symbol blocks of the different active users, and $\mathrm{C}[i]:=$ $\left[\mathbf{c}^{1}[i], \ldots, \mathbf{c}^{M}[i]\right]$ collects the composite code vectors of the different active users. The block spreading operation in (3) can be viewed as classical symbol spreading, where every user's information symbols on the different subcarriers are spread along the time axis, using the same spreading code. Furthermore, by choosing $Q$ sufficiently high, each subcarrier experiences frequency-flat fading, such that the orthogonality among users is preserved on every subcarrier, even after propagation through a frequency-selective channel. Consequently, as will become apparent in Section 2.2, block spreading enables MUI-resilient reception and thus effectively deals with the MUI. Subsequently, the $Q \times Q$ IFFT matrix $\mathbf{F}_{Q}^{H}$ transforms the FD chip block sequence $\tilde{\mathbf{x}}[n]$ into the time-domain (TD) chip block sequence: $\mathbf{x}[n]=\mathbf{F}_{Q}^{H} \cdot \tilde{\mathbf{x}}[n]$.

The third operation involves the addition of transmit redundancy. Specifically, the $K \times Q$ transmit matrix $\mathrm{T}$, with $K$ the transmitted block length, $K \geq Q$, adds some redundancy to the chip blocks $\mathbf{x}[n]$, that is, $\mathbf{u}[n]:=\mathbf{T} \cdot \mathbf{x}[n]$. As will be clarified later, this transmit redundancy copes with the time-dispersive effect of multipath propagation, and enables low-complexity equalization at the receiver. Finally, the resulting transmitted chip block sequence $\mathbf{u}[n]$ is parallelto-serial converted into the corresponding scalar sequence $[u[n K], \ldots, u[(n+1) K-1]]^{T}:=\mathbf{u}[n]$, and transmitted over the air at a rate $1 / T_{c}$. By analyzing the rates of the different transmitter blocks in Figure 1, it is clear that the channel symbol rate, $R_{s}$, relates to the chip rate, $R_{c}$, through $R_{s}=(B / K)(1 / N) R_{c}$.

From a bandwidth utilization point of view, the BS transmits $B$ information symbols to each of the $M$ users, using $N K=N(Q+L)=N(B+2 L)$ transmitted chips, where the overhead of $2 L$ stems from the $(B+L) \times B$ redundant linear precoder, $\Theta$, which guarantees symbol detection, and the length- $L \mathrm{CP}$, which is common to all users and removes interblock interference (IBI). Therefore, the bandwidth efficiency of our transceiver supporting $M$ users can be calculated as

$$
\epsilon_{\mathrm{MCBS}-\mathrm{CDMA}}=\frac{M B}{N K}=\frac{M B}{N(B+2 L)} \leq 1 .
$$

Clearly, as the number of users approaches its maximum value, that is, $M=N$, the bandwidth efficiency also converges to its maximum value, $\bar{\epsilon}_{\mathrm{MCBS}-\mathrm{CDMA}}=B /(B+2 L)$.

\subsection{MUI-resilient reception with MCBS-CDMA}

Adopting a discrete-time baseband equivalent model, the synchronized and chip-sampled received signal is a channeldistorted version of the transmitted signal, and can be written as

$$
v[n]=\sum_{l=0}^{L_{c}} h[l] u[n-l]+w[n],
$$

where $h[l]$ is the chip-sampled FIR channel that models the frequency-selective multipath propagation between the transmitter and the receiver including the effect of transmit and receive filters, $L_{c}$ is the order of $h[l]$, and $w[n]$ denotes the additive Gaussian noise, which we assume to be white with variance $\sigma_{w}^{2}$. Furthermore, we define $L$ as a known upper bound on the channel order $L \geq L_{c}$, which can be well approximated by $L \approx\left\lfloor\tau_{\max } / T_{c}\right\rfloor+1$, where $\tau_{\max }$ is the maximum excess delay within the given propagation environment.

The block diagram in Figure 2 describes the reception scheme for the MS of interest (which we assume to be the $m$ th one), which transforms the received sequence $v[n]$ into an estimate of the desired user's data symbol sequence $\hat{s}^{m}[i]$. Assuming perfect chip and block synchronization, the received sequence $v[n]$ is serial-to-parallel converted into its corresponding block sequence $\mathbf{v}[n]:=[v[n K], \ldots, v[(n+1) K-$ $1]]^{T}$. From the scalar input/output relationship in (5), we can derive the corresponding block input/output relationship:

$$
\mathbf{v}[n]=\mathbf{H}[0] \cdot \mathbf{u}[n]+\mathbf{H}[1] \cdot \mathbf{u}[n-1]+\mathbf{w}[n],
$$

where $\mathbf{w}[n]:=[w[n K], \ldots, w[(n+1) K-1]]^{T}$ is the noise block sequence, $\mathbf{H}[0]$ is a $K \times K$ lower triangular Toeplitz matrix with entries $[\mathbf{H}[0]]_{p, q}=h[p-q]$, and $\mathbf{H}[1]$ is a $K \times K$ upper triangular Toeplitz matrix with entries $[\mathbf{H}[1]]_{p, q}=$ $h[K+p-q]$ (see, e.g., [11] for a detailed derivation of the single-user case). The time-dispersive nature of multipath propagation gives rise to so-called IBI between successive blocks, which is modelled by the second term in (6). The $Q \times K$ receive matrix $\mathbf{R}$ again removes the redundancy from the blocks $\mathbf{v}[n]: \mathbf{y}[n]:=\mathbf{R} \cdot \mathbf{v}[n]$. The purpose of the trans$\mathrm{mit} /$ receive pair $(\mathbf{T}, \mathbf{R})$ is twofold. First, it allows for simple block-by-block processing by removing the IBI. Second, it enables low-complexity FD equalization by making the linear channel convolution appear circulant to the received block. 
To guarantee perfect IBI removal, the pair $(\mathbf{T}, \mathbf{R})$ should satisfy the following condition [11]:

$$
\mathbf{R} \cdot \mathbf{H}[1] \cdot \mathbf{T}=\mathbf{0} .
$$

To enable circulant channel convolution, the resulting channel matrix $\dot{\mathbf{H}}:=\mathbf{R} \cdot \mathbf{H}[0] \cdot \mathbf{T}$ should be circulant. In this way, we obtain a simplified block input/output relationship in the TD:

$$
\mathbf{y}[n]=\dot{\mathbf{H}} \cdot \mathbf{x}[n]+\mathbf{z}[n]
$$

where $\mathbf{z}[n]:=\mathbf{R} \cdot \mathbf{w}[n]$ is the corresponding noise block sequence. In general, two options for the pair $(\mathbf{T}, \mathbf{R})$ exist that satisfy the above conditions. The first option corresponds to $\mathrm{CP}$ in classical OFDM systems [23], and boils down to choosing $K=Q+L$, and selecting

$$
\mathbf{T}=\mathbf{T}_{c p}:=\left[\mathbf{I}_{c p}^{T}, \mathbf{I}_{Q}^{T}\right]^{T}, \quad \mathbf{R}=\mathbf{R}_{c p}:=\left[\mathbf{0}_{Q \times L}, \mathbf{I}_{Q}\right]
$$

where $\mathbf{I}_{c p}$ consists of the last $L$ rows of $\mathbf{I}_{Q}$. The circulant property is enforced at the transmitter by adding a cyclic prefix of length $L$ to each block. Indeed, premultiplying a vector with $\mathrm{T}_{c p}$ copies its last $L$ entries and pastes them to its top. The IBI is removed at the receiver by discarding the cyclic prefix of each received block. Indeed, premultiplying a vector with $\mathbf{R}_{c p}$ deletes its first $L$ entries and thus satisfies (7).

The second option corresponds to $\mathrm{ZP}$, and boils down to setting $K=Q+L$, and selecting

$$
\mathbf{T}=\mathbf{T}_{z p}:=\left[\mathbf{I}_{Q}^{T}, \mathbf{0}_{Q \times L}^{T}\right]^{T}, \quad \mathbf{R}=\mathbf{R}_{z p}:=\left[\mathbf{I}_{Q}, \mathbf{I}_{z p}\right],
$$

where $\mathbf{I}_{z p}$ is formed by the first $L$ columns of $\mathbf{I}_{Q}$. Unlike classical OFDM systems, here the IBI is entirely dealt with at the transmitter. Indeed, premultiplying a vector with $\mathbf{T}_{z p}$ pads $L$ trailing zeros to its bottom and thus satisfies (7). The circulant property is enforced at the receiver by time-aliasing each received block. Indeed, premultiplying a vector with $\mathbf{R}_{z p}$ adds its last $L$ entries to its first $L$ entries.

Referring back to (8), circulant matrices possess a nice property that enables simple per-tone equalization in the FD.

Property 1. Circulant matrices can be diagonalized by FFT operations [24]

$$
\dot{\mathbf{H}}=\mathbf{F}_{Q}^{H} \cdot \tilde{\mathbf{H}} \cdot \mathbf{F}_{Q},
$$

with $\tilde{\mathbf{H}}:=\operatorname{diag}(\tilde{\mathbf{h}}), \quad \tilde{\mathbf{h}} \quad:=\left[H\left(e^{j 0}\right), H\left(e^{j(2 \pi / Q)}\right), \ldots\right.$, $\left.H\left(e^{j(2 \pi / Q)(Q-1)}\right)\right]$ the FD channel response evaluated on the FFT grid, $H(z):=\sum_{l=0}^{L} h[l] z^{-l}$ the $z$-transform of $h[l]$, and $\mathrm{F}_{Q}$ the $Q \times Q$ FFT matrix.

Aiming at low-complexity FD processing, we transform $\mathbf{y}[n]$ into the FD by defining $\tilde{\mathbf{y}}[n]:=\mathbf{F}_{Q} \cdot \mathbf{y}[n]$. Relying on Property 1, this leads to the following FD block input/output relationship:

$$
\tilde{\mathbf{y}}[n]=\tilde{\mathbf{H}} \cdot \tilde{\mathbf{x}}[n]+\tilde{\mathbf{z}}[n],
$$

where $\tilde{\mathbf{z}}[n]:=\mathbf{F}_{Q} \cdot \mathbf{z}[n]$ is the corresponding FD noise block sequence. Collecting $N$ consecutive chip blocks $\tilde{\mathbf{y}}[n]$ into $\tilde{\mathbf{Y}}[i]:=[\tilde{\mathbf{Y}}[i N], \ldots, \tilde{\mathbf{Y}}[(i+1) N-1]]$, defining $\tilde{\mathbf{X}}[i]$ and $\tilde{\mathbf{Z}}[i]$ in a similar manner as $\tilde{\mathbf{Y}}[i]$, and exploiting (3), we obtain the symbol block level equivalent of (12), that is,

$$
\tilde{\mathbf{Y}}[i]=\tilde{\mathbf{H}} \cdot \tilde{\mathbf{S}}[i] \cdot \mathbf{C}[i]^{T}+\tilde{\mathbf{Z}}[i] .
$$

By inspecting (13), we can conclude that our transceiver preserves the orthogonality among users, even after propagation through a (possibly unknown) frequency-selective multipath channel. This property allows for deterministic MUI elimination through low-complexity code-matched filtering. Indeed, by block despreading (13) with the desired user's composite code vector $\mathbf{c}^{m}[i]$ (we assume the $m$ th user to be the desired one), we obtain

$$
\tilde{\mathbf{Y}}^{m}[i]:=\tilde{\mathbf{Y}}[i] \cdot \mathbf{c}^{m}[i]^{*}=\tilde{\mathbf{H}} \cdot \boldsymbol{\Theta} \cdot \mathbf{s}^{m}[i]+\tilde{\mathbf{z}}^{m}[i],
$$

where $\tilde{\mathbf{z}}^{m}[i]:=\tilde{\mathbf{Z}}[i] \cdot \mathbf{c}^{m}[i]^{*}$ is the corresponding noise block sequence. Our transceiver successfully converts (through block despreading) a multiuser detection problem into an equivalent but simpler single-user equalization problem. Moreover, the operation of block despreading preserves ML optimality, since it does not incur any information loss in the Shannon sense regarding the desired user's symbol block $\mathbf{s}^{m}[i]$.

In the above discussion, our main focus was on the downlink problem, which is simpler in nature than the uplink problem, since the different user signals experience the same multipath channel, time offset, and carrier frequency offset. In theory, the same signal design is also feasible in the uplink. Assuming perfect time and frequency synchronization between the different users and the BS, it can be shown that the orthogonality among users is still preserved, even if the user signals now propagate through a different multipath channel. In practice, perfect time and frequency synchronization cannot be guaranteed, since the user signals experience a different time offset and carrier frequency offset, with respect to the BS. Furthermore, the BS receiver can only compensate for a certain user's synchronization mismatches after this user's signal has been separated from the received multiuser mixture. Otherwise, a compensation for that particular user would affect all other users too. However, since the proposed block spreading scheme relies on the orthogonality preservation property, which requires perfect time and frequency synchronization, the synchronization mismatches would have introduced irreducible distortion at that point already. Therefore, in contrast with the downlink, which can rely on existing single-user schemes, a new scheme is needed in the uplink, in which each user estimates its synchronization mismatches with respect to the BS and compensates these before transmission, which we refer to as presynchronization. Only the small residual mismatches that remain after pre-synchronization should be compensated after separation, which we refer to as postsynchronization. 


\subsection{Single-user equalization for MCBS-CDMA}

After successful elimination of the MUI, we still need to detect the desired user's symbol block $\mathbf{s}^{m}[i]$ from (14). Ignoring, for the moment, the presence of $\Theta$ (or, equivalently, setting $Q=B$ and selecting $\boldsymbol{\Theta}=\mathbf{I}_{Q}$ ), this requires $\tilde{\mathbf{H}}$ to have full column rank $Q$. Unfortunately, this condition only holds for channels that do not invoke any zero diagonal entries in $\tilde{\mathbf{H}}$. In other words, if the MS experiences a deep channel fade on a particular tone (corresponding to a zero diagonal entry in $\tilde{\mathbf{H}}$ ), the information symbol on that tone cannot be recovered. To guarantee symbol detectability of the $B$ symbols in $\mathbf{s}^{m}[i]$, regardless of the symbol constellation, we thus need to design the precoder $\Theta$ such that

$$
\operatorname{rank}(\tilde{\mathbf{H}} \cdot \boldsymbol{\Theta})=B,
$$

irrespective of the underlying channel realization [11]. Since an FIR channel of order $L$ can invoke at most $L$ zero diagonal entries in $\tilde{\mathbf{H}}$, this requires any $Q-L=B$ rows of $\Theta$ to be linearly independent.

In $[21,22]$, two classes of precoders have been constructed that satisfy this condition and thus guarantee symbol detectability or, equivalently, enable full frequencydiversity gain; namely, the Vandermonde precoders and the real cosine precoders. The $Q \times B$ complex Vandermonde precoder is defined by $[\boldsymbol{\Theta}(\boldsymbol{\rho})]_{q, b}=\rho_{q}^{b}$, where $\boldsymbol{\rho}:=$ $\left[\rho_{0}, \ldots, \rho_{Q-1}\right]^{T}$, and the $\rho_{q}$ 's, with $q=0, \ldots, Q-1$, are $Q$ complex points, such that $\rho_{q} \neq \rho_{q^{\prime}}$ for all $q \neq q^{\prime}$. A special case of the general Vandermonde precoder is a truncated FFT matrix, defined by choosing $\rho_{q}=\exp (-j 2 \pi q / Q)$. The $Q \times B$ real cosine precoder is defined by $[\Theta(\phi)]_{q, b}=$ $\cos (b+1 / 2) \phi_{q}$, where $\phi:=\left[\phi_{0}, \ldots, \phi_{Q-1}\right]^{T}$, and the $\phi_{q}$ 's, with $q=0, \ldots, Q-1$, are $Q$ real points, such that $\phi_{q} \neq(2 k+1) \pi$ and $\phi_{q} \pm \phi_{q}^{\prime} \neq 2 k \pi$ for all $q \neq q^{\prime}$ and $k$ integer. A special case of the general cosine precoder is a truncated discrete cosine transform (DCT) matrix, defined by choosing $\phi_{q}=q \pi / Q$.

\section{EQUALIZATION OPTIONS}

In this section, we discuss different options to perform equalization and decoding of the linear precoding, either jointly or separately, under the assumption of perfect CSI at the receiver. These options allow to trade-off performance versus complexity, ranging from optimal ML detection with exponential complexity to linear and decision-directed detection with linear complexity. To evaluate the complexity, we distinguish between the initialization phase, when the equalizers are calculated based on the channel knowledge, and the data processing phase, when the received data is actually processed. The rate of the former is related to the channel's fading rate, whereas that of the latter is executed continuously at the symbol block rate. By analyzing the rate of the different receiver blocks in Figure 2, it is clear that the equalizer operates at a rate which is $B$ times lower than the symbol rate that is, $R_{\mathrm{eq}}=R_{s} / B$.

This section is organized as follows. Section 3.1 investigates ML detection. Section 3.2 studies joint linear equalization and decoding, whereas Section 3.3 introduces joint deci-
TABLE 1: Complexity of ML.

\begin{tabular}{l|c}
\hline & Data processing \\
\hline Multiplications & $Q \mathcal{C}^{B}$ \\
Additions & $Q \frac{2 \mathcal{C}^{B+1}-\mathcal{C}^{B}-1}{\mathcal{C}-1}-\mathcal{C}^{B}$ \\
Data transfers & $3 Q \frac{2 \mathcal{C}^{B+1}-\mathcal{C}^{B}-1}{\mathcal{C}}-1+2 Q \mathcal{C}^{B}-3$ \\
\hline
\end{tabular}

sion feedback equalization and decoding. Finally, Section 3.4 proposes separate linear equalization and decoding.

\section{1. $M L$ detection}

The ML algorithm is optimal in an ML sense but has a very high complexity. Amongst all possible transmitted blocks, it retains the one that maximizes the likelihood function or, equivalently, minimizes the Euclidean distance:

$$
\underline{\hat{\mathbf{s}}}^{m}[i]=\arg \min _{\mathbf{s}^{m}[i] \in \mathcal{S}}\left\|\tilde{\mathbf{y}}^{m}[i]-\tilde{\mathbf{H}} \cdot \boldsymbol{\Theta} \cdot \mathbf{s}^{m}[i]\right\|^{2} .
$$

In other words, the ML metric is given by the Euclidean distance between the actual received block and the block that would have been received if a particular symbol block had been transmitted in a noiseless environment. The number of possible transmit vectors in $\&$ is the cardinality of $\&$, that is, $|\&|=\mathcal{C}^{B}$, with $\mathcal{C}$ the constellation size. Consequently, the number of points to inspect grows exponentially with the initial block length $B$.

The ML algorithm does not require an initialization phase. During the data processing phase, the ML algorithm calculates the Euclidean distance metric of (16), for all possible transmit vectors $\mathbf{s}^{m}[i]$. To lower the complexity, a treelike implementation avoids frequent recalculation of common subexpressions. Table 1 summarizes the complexity of the ML algorithm in terms of complex multiplications, additions, and data transfers. The overall complexity is $\mathcal{O}\left(Q \mathcal{C}^{B}\right)$ during data processing. Hence, this algorithm is only feasible for a small block length $B$ and a small constellation size $\mathcal{C}$.

\subsection{Joint linear equalization and decoding}

Linear equalizers that perform joint equalization and decoding combine a low complexity with medium performance. A first possibility is to apply a zero-forcing block linear equalizer (ZF-BLE) [25]

$$
\mathbf{G}_{\mathrm{ZF}}=\left(\Theta^{H} \cdot \tilde{\mathbf{H}}^{H} \cdot \tilde{\mathbf{H}} \cdot \boldsymbol{\Theta}\right)^{-1} \cdot \Theta^{H} \cdot \tilde{\mathbf{H}}^{H},
$$

which completely eliminates the ISI, irrespective of the noise level. A second possibility is to apply a minimum meansquare-error block linear equalizer (MMSE-BLE) [25]

$$
\mathbf{G}_{\mathrm{MMSE}}=\left(\boldsymbol{\Theta}^{H} \cdot \tilde{\mathbf{H}}^{H} \cdot \tilde{\mathbf{H}} \cdot \boldsymbol{\Theta}+\frac{\sigma_{w}^{2}}{\sigma_{s}^{2}} \mathbf{I}_{B}\right)^{-1} \cdot \boldsymbol{\Theta}^{H} \cdot \tilde{\mathbf{H}}^{H},
$$

which minimizes the MSE between the actual transmitted symbol block and its estimate. Here, $\sigma_{w}^{2}$ and $\sigma_{s}^{2}$ are the noise variance and the information symbol variance, respectively. 
TABLe 2: Complexity of ZF-BLE.

\begin{tabular}{l|cc}
\hline & Initialization & Data processing \\
\hline Multiplications & $\frac{B^{3} Q}{3}+3 B^{2} Q+\frac{13}{6} B Q$ & $B Q$ \\
Additions & $\frac{B^{3} Q}{3}+3 B^{2} Q-\frac{5}{6} B Q-B^{2}$ & $B Q-B$ \\
Data transfers & $2 B^{3} Q+21 B^{2} Q+7 B Q-3 B^{2}$ & $6 B Q-3 B$ \\
\hline
\end{tabular}

TABLe 3: Complexity of MMSE-BLE.

\begin{tabular}{l|cc}
\hline & Initialization & Data processing \\
\hline Multiplications & $\frac{B^{3} Q}{6}+\frac{5}{2} B^{2} Q+\frac{7}{3} B Q+1$ & $B Q$ \\
Additions & $\frac{5}{2} B^{2} Q-\frac{B Q}{2}-B^{2}+B$ & $B Q-B$ \\
Data transfers & $\frac{B^{3} Q}{2}+15 B^{2} Q+\frac{11}{2} B Q-3 B^{2}+3 B+3$ & $6 B Q-3 B$ \\
\hline
\end{tabular}

During the initialization phase, $\mathbf{G}_{\mathrm{ZF}}$ and $\mathbf{G}_{\mathrm{MMSE}}$ can be computed from the set of multiple linear systems, implicitly shown in (17) and (18), respectively. For the ZF-BLE, the solution of each linear system can be found using the $L U$ decomposition, which relies on Gauss elimination with partial pivoting [24]. For the MMSE-BLE, each linear system can be solved based on the $L D L^{H}$ decomposition (instead of the $L U$ decomposition), which relies on Gauss elimination without pivoting [24]. During the data processing phase, the equalizers $\mathbf{G}_{\mathrm{ZF}}$ and $\mathbf{G}_{\mathrm{MMSE}}$ are applied to the received block $\tilde{\mathbf{y}}^{m}[i]$. Tables 2 and 3 summarize the complexity of the ZF- and the MMSE-BLE, respectively, in terms of complex multiplications, additions, and data transfers. In both cases, the overall complexity is $\mathcal{O}\left(B^{3} Q\right)$ during initialization and $\mathcal{O}(B Q)$ during data processing.

\subsection{Joint decision feedback equalization and decoding}

The class of nonlinear equalizers that perform joint decision feedback equalization and decoding lies in between the former categories, both in terms of performance and in complexity. The block decision feedback equalizers (BDFEs) consist of a feedforward section, represented by the matrix $\mathbf{W}$, and a feedback section, represented by the matrix $\mathbf{B}[26,27]$ :

$$
\underline{\hat{\mathbf{s}}}^{m}[i]=\operatorname{slice}\left[\mathbf{W} \cdot \tilde{\mathbf{y}}^{m}[i]-\mathbf{B} \cdot \underline{\hat{\mathbf{s}}}^{m}[i]\right] .
$$

The feedforward and feedback sections can be designed according to a ZF or MMSE criterium. In either case, $\mathbf{B}$ should be a strictly upper or lower triangular matrix with zero diagonal entries, in order to feedback decisions in a causal way. To design the decision feedback counterpart of the ZFBLE, we compute the Cholesky decomposition of the matrix $\boldsymbol{\Theta}^{H} \cdot \tilde{\mathbf{H}}^{H} \cdot \tilde{\mathbf{H}} \cdot \boldsymbol{\Theta}$ in $(17)$, that is,

$$
\Theta^{H} \cdot \tilde{\mathbf{H}}^{H} \cdot \tilde{\mathbf{H}} \cdot \boldsymbol{\Theta}=\left(\boldsymbol{\Sigma}_{1} \cdot \mathbf{U}_{1}\right)^{H} \cdot \boldsymbol{\Sigma}_{1} \cdot \mathbf{U}_{1},
$$

where $\mathbf{U}_{1}$ is an upper triangular matrix with ones along the diagonal and $\Sigma_{1}$ is a diagonal matrix with real entries. The ZF-BDFE then follows from

$$
\begin{gathered}
\mathbf{W}_{\mathrm{ZF}}=\mathbf{U}_{1} \cdot \mathbf{G}_{\mathrm{ZF}}=\boldsymbol{\Sigma}_{1}^{-1} \cdot\left(\mathbf{U}_{1}^{H} \cdot \boldsymbol{\Sigma}_{1}\right)^{-1} \cdot \boldsymbol{\Theta}^{H} \cdot \tilde{\mathbf{H}}^{H}, \\
\mathbf{B}_{\mathrm{ZF}}=\mathbf{U}_{1}-\mathbf{I}_{B} .
\end{gathered}
$$

The linear feedforward section $\mathbf{W}_{\mathrm{ZF}}$ suppresses the ISI originating from "future" symbols, the so-called precursor ISI, whereas the nonlinear feedback section $\mathbf{B}_{\mathrm{ZF}}$ eliminates the ISI originating from "past" symbols, the so-called postcursor ISI.

Likewise, to design the decision feedback counterpart of the MMSE-BLE, we compute the Cholesky decomposition of the matrix $\boldsymbol{\Theta}^{H} \cdot \tilde{\mathbf{H}}^{H} \cdot \tilde{\mathbf{H}} \cdot \boldsymbol{\Theta}+\left(\sigma_{w}^{2} / \sigma_{s}^{2}\right) \mathbf{I}_{B}$ in $(18)$, that is,

$$
\boldsymbol{\Theta}^{H} \cdot \tilde{\mathbf{H}}^{H} \cdot \tilde{\mathbf{H}} \cdot \boldsymbol{\Theta}+\frac{\sigma_{w}^{2}}{\sigma_{s}^{2}} \mathbf{I}_{B}=\left(\boldsymbol{\Sigma}_{2} \cdot \mathbf{U}_{2}\right)^{H} \cdot \boldsymbol{\Sigma}_{2} \cdot \mathbf{U}_{2},
$$

where $\mathbf{U}_{2}$ is an upper triangular matrix with ones along the diagonal, and $\Sigma_{2}$ is a diagonal matrix with real entries. The MMSE-BDFE can then be calculated as

$$
\begin{gathered}
\mathbf{W}_{\mathrm{MMSE}}=\mathbf{U}_{2} \cdot \mathbf{G}_{\mathrm{MMSE}}=\boldsymbol{\Sigma}_{2}^{-1} \cdot\left(\mathbf{U}_{2}^{H} \cdot \boldsymbol{\Sigma}_{2}\right)^{-1} \cdot \boldsymbol{\Theta}^{H} \cdot \tilde{\mathbf{H}}^{H}, \\
\mathbf{B}_{\mathrm{MMSE}}=\mathbf{U}_{2}-\mathbf{I}_{B} .
\end{gathered}
$$

During the initialization phase, the feedforward and feedback filters of the ZF- and MMSE-BDFE are computed based on (21) and (23), respectively, relying on the Cholesky decomposition [24]. During the data processing phase, the received data is first filtered with the feedforward filter, W, and then fed back with the feedback filter, B, according to (19). Tables 4 and 5 summarize the complexity of the ZF- and MMSE-BDFE, respectively, in terms of complex multiplications, additions, and data transfers. In both cases, the overall complexity is $\mathcal{O}\left(B^{3} Q\right)$ during initialization and $\mathcal{O}(B Q)$ during data processing. Hence, the nonlinear BDFEs involve the same order of complexity as their linear counterparts. 
TABLE 4: Complexity of ZF-BDFE.

\begin{tabular}{l|cc}
\hline & Initialization & Data processing \\
\hline Multiplications & $\frac{B^{3} Q}{3}+4 B^{2} Q+\frac{B^{3}}{6}+\frac{13}{6} B Q+\frac{B^{2}}{2}+\frac{B}{3}$ & $B Q+B^{2}$ \\
Additions & $\frac{B^{3} Q}{3}+4 B^{2} Q+\frac{B^{3}}{6}-\frac{11}{6} B Q-B^{2}+\frac{5}{6} B$ & $B Q+B^{2}-B$ \\
Data transfers & $2 B^{3} Q+27 B^{2} Q+B^{3}+4 B Q-B^{2}+4 B$ & $6 B Q+6 B^{2}-3 B$ \\
\hline
\end{tabular}

TABLe 5: Complexity of MMSE-BDFE.

\begin{tabular}{l|cc}
\hline & Initialization & Data processing \\
\hline Multiplications & $\frac{B^{3} Q}{6}+\frac{7}{2} B^{2} Q+\frac{B^{3}}{6}+\frac{7}{3} B Q+\frac{B^{2}}{2}+\frac{B}{3}+1$ & $B Q+B^{2}$ \\
Additions & $\frac{7}{2} B^{2} Q+\frac{B^{3}}{6}-\frac{3}{2} B Q-B^{2}+\frac{11}{6} B$ & $B Q+B^{2}-B$ \\
Data transfers & $\frac{B^{3} Q}{2}+21 B^{2} Q+B^{3}+\frac{5}{2} B Q-B^{2}+7 B+3$ & $6 B Q+6 B^{2}-3 B$ \\
\hline
\end{tabular}

\subsection{Separate linear equalization and decoding}

Previously, we have only considered joint equalization and decoding of the linear precoding. However, in order to even further reduce the complexity with respect to the block linear equalizers of Section 3.2, equalization and decoding can be performed separately as well:

$$
\hat{\mathbf{s}}^{m}[i]=\boldsymbol{\Theta}^{H} \cdot \tilde{\mathbf{G}} \cdot \tilde{\mathbf{y}}^{m}[i],
$$

for which we rely on the para-unitary property of $\Theta$. Here, $\tilde{\mathbf{G}}$ performs per-tone linear equalization (PT-LE) only, and tries to restore $\widetilde{\mathbf{s}}^{m}[i]$, whereas $\boldsymbol{\Theta}^{H}$ subsequently performs linear decoding only, and tries to restore $\mathbf{s}^{m}[i]$.

The ZF-per-tone linear equalizer (PT-LE), which can be expressed as

$$
\tilde{\mathbf{G}}_{\mathrm{ZF}}=\left(\tilde{\mathbf{H}}^{H} \cdot \tilde{\mathbf{H}}\right)^{-1} \cdot \tilde{\mathbf{H}}^{H},
$$

perfectly removes the amplitude and phase distortion on every tone, irrespective of the noise level.

The MMSE-PT-LE, which balances amplitude and phase distortion with noise enhancement on every tone, can be expressed as

$$
\tilde{\mathbf{G}}_{\mathrm{MMSE}}=\left(\tilde{\mathbf{H}}^{H} \cdot \tilde{\mathbf{H}}+\sigma_{w}^{2} \mathbf{R}_{\tilde{\mathbf{s}}}^{-1}\right)^{-1} \cdot \tilde{\mathbf{H}}^{H},
$$

where $\mathbf{R}_{\tilde{\mathbf{s}}}:=\mathrm{E}\left\{\tilde{\mathbf{s}}^{m}[i] \cdot \tilde{\mathbf{s}}^{m}[i]^{H}\right\}=\sigma_{s}^{2} \Theta \cdot \Theta^{H}$ is the covariance matrix of $\tilde{\mathbf{s}}^{m}[i]$. The MMSE equalizer only decouples into $Q$ parallel and independent single-tap equalizers, if we neglect the color in the precoded symbols, that is, $\mathbf{R}_{\tilde{s}} \approx \sigma_{s}^{2} \mathbf{I}_{Q}$.

During the initialization phase, $\tilde{\mathbf{G}}_{\mathrm{ZF}}$ and $\tilde{\mathbf{G}}_{\mathrm{MMSE}}$ are calculated from (25) and (26), respectively, where the matrix inversion reduces to $Q$ parallel scalar divisions. During the data processing phase, the received data is separately equalized and decoded, according to (24). Furthermore, the linear decoding step relies on implementation efficient IDCT or IFFT operations. Tables 6 and 7 summarize the complexity of the ZF- and MMSE-PT-LE, respectively, in terms of com- plex multiplications, additions, and data transfers. In both cases, the overall complexity is $\mathcal{O}(Q)$ during initialization and $\mathcal{O}\left(Q \log _{2}(Q)\right)$ during data processing.

\section{EXTENSION TO MULTIPLE ANTENNAS}

As shown in Sections 2 and 3, MCBS-CDMA successfully addresses the challenges of broadband cellular downlink communications. However, the spectral efficiency of singleantenna MCBS-CDMA is still limited by the received signalto-noise ratio (SNR) and cannot be further improved by traditional communication techniques. As opposed to singleantenna systems, MIMO systems that deploy $N_{T}$ transmit and $N_{R}$ receive antennas enable an $N_{\min }$-fold capacity increase in rich scattering environments, where $N_{\min }=$ $\min \left\{N_{T}, N_{R}\right\}$ is called the multiplexing gain $[28,29,30]$. Besides the time, frequency, and code dimensions, MIMO systems create an extra spatial dimension that allows to increase the spectral efficiency and/or to improve the performance. On the one hand, space-division multiplexing (SDM) techniques achieve high spectral efficiency by exploiting the spatial multiplexing gain [31] (see also [32]). On the other hand, space-time coding (STC) techniques achieve high qualityof-service (QoS) by exploiting diversity and coding gains $[33,34,35]$. Besides the leverages they offer, MIMO systems also sharpen the challenges of broadband cellular downlink communications. First, time dispersion and ISI are now caused by $N_{T} N_{R}$ frequency-selective multipath fading channels instead of just 1 . Second, MUI originates from $N_{T} M$ sources instead of just $M$. Third, the presence of multiple antennas seriously impairs a low-complexity implementation of the MS. To tackle these challenges, we will demonstrate the synergy between our MCBS-CDMA waveform and MIMO signal processing. In particular, we focus on a space-time block-coded (STBC) MCBS-CDMA transmission, but the general principles apply equally well to a space-time trellis coded or a space-division multiplexed MCBS-CDMA transmission. 
TABLE 6: Complexity of ZF-PT-LE.

\begin{tabular}{l|cc}
\hline & Initialization & Data processing \\
\hline Multiplications & $2 Q$ & $Q\left(\frac{1}{2} \log _{2}(Q)+1\right)$ \\
Additions & - & $Q \log _{2}(Q)$ \\
Data transfers & $6 Q$ & $3 Q\left(\frac{3}{2} \log _{2}(Q)+1\right)$ \\
\hline
\end{tabular}

TABle 7: Complexity of MMSE-PT-LE.

\begin{tabular}{l|cc}
\hline & Initialization & Data processing \\
\hline Multiplications & $2 Q+1$ & $Q\left(\frac{1}{2} \log _{2}(Q)+1\right)$ \\
Additions & $Q$ & $Q \log _{2}(Q)$ \\
Data transfers & $9 Q+3$ & $3 Q\left(\frac{3}{2} \log _{2}(Q)+1\right)$ \\
\hline
\end{tabular}

This section is organized as follows. Section 4.1 details the STBC MCBS-CDMA transmission scheme for the case of $N_{T}=2$ transmit antennas. Section 4.2 demonstrates how the user orthogonality preservation property of MCBS-CDMA translates to the MIMO case, which allows to convert a difficult multiuser MIMO detection problem into an equivalent but simpler single-user MIMO equalization problem. Finally, Section 4.3 explains how space-time decoding and equalization can then be performed for each user separately.

\subsection{Space-time block-coded MCBS-CDMA transmission}

The block diagram in Figure 3 describes the STBC MCBSCDMA downlink transmission scheme (where only the $m$ th user is explicitly shown), that transforms the $M$ user data symbol sequences $\left\{s^{m}[i]\right\}_{m=1}^{M}$ into $N_{T}$ ST coded multiuser chip sequences $\left\{u_{n_{t}}[n]\right\}_{n_{t}=1}^{N_{T}}$ with a rate $1 / T_{c}$. For conciseness, we limit ourselves to the case of $N_{T}=2$ transmit antennas with rate $R=1$ space-time block codes. Note, however, that the proposed technique can be easily extended to the case of $N_{T}>2$ transmit antennas with $R=1 / 2$ space-time block codes, by resorting to the generalized orthogonal designs of [35]. As for the single-antenna case, the information symbols are first grouped into blocks of $B$ symbols and linearly precoded. Unlike the traditional approach of performing ST encoding at the scalar symbol level, we perform ST encoding at the symbol block level; this was also done in, for example, [36]. Out ST encoder operates in the FD and takes two consecutive symbol blocks $\left\{\tilde{\mathbf{s}}^{m}[2 i], \tilde{\mathbf{s}}^{m}[2 i+1]\right\}$ to output the following $2 Q \times 2$ matrix of ST coded symbol blocks:

$$
\left[\begin{array}{ll}
\overline{\mathbf{s}}_{1}^{m}[2 i] & \overline{\mathbf{s}}_{1}^{m}[2 i+1] \\
\overline{\mathbf{s}}_{2}^{m}[2 i] & \overline{\mathbf{s}}_{2}^{m}[2 i+1]
\end{array}\right]=\left[\begin{array}{cc}
\tilde{\mathbf{s}}^{m}[2 i] & -\tilde{\mathbf{s}}^{m}[2 i+1]^{*} \\
\tilde{\mathbf{s}}^{m}[2 i+1] & \tilde{\mathbf{s}}^{m}[2 i]^{*}
\end{array}\right] .
$$

At each time interval $i$, the ST coded symbol blocks $\overline{\mathbf{s}}_{1}^{m}[i]$ and $\overline{\mathbf{s}}_{2}^{m}[i]$ are forwarded to the first and the second transmit antenna, respectively. From (27), we can easily verify that the transmitted symbol block at time instant $2 i+1$ from one antenna is the conjugate of the transmitted symbol block at time instant $2 i$ from the other antenna (with a possible sign change). This corresponds to a per-tone implementation of the classical Alamouti scheme for frequency-flat fading channels [34]. As we will show later, this property allows for deterministic transmit stream separation at the receiver.

After ST encoding, the resulting symbol block sequences $\left\{\overline{\mathbf{s}}_{n_{t}}^{m}[i]\right\}_{n_{t}=1}^{N_{T}}$ are block-spread and code-division multiplexed with those of the other users:

$$
\tilde{\mathbf{x}}_{n_{t}}[n]=\sum_{m=1}^{M} \overline{\mathbf{s}}_{n_{t}}^{m}[i] c^{m}[n], \quad n=i N+n^{\prime} .
$$

At this point, it is important to note that each of the $N_{T}$ parallel block sequences are block spread by the same composite code sequence $c^{m}[n]$, guaranteeing an efficient utilization of the available code space. As will become apparent later, this property allows for deterministic user separation at every receive antenna. After IFFT transformation and the addition of some form of transmit redundancy

$$
\mathbf{u}_{n_{t}}[n]=\mathbf{T} \cdot \mathbf{F}_{Q}^{H} \cdot \tilde{\mathbf{x}}_{n_{t}}[n],
$$

the corresponding scalar sequences $\left\{u_{n_{t}}[n]\right\}_{n_{t}=1}^{N_{T}}$ are transmitted over the air at a rate $1 / T_{c}$.

\subsection{MUI-resilient MIMO reception}

The block diagram in Figure 4 describes the reception scheme for the MS of interest, which transforms the different received sequences $\left\{v_{n_{r}}[n]\right\}_{n_{r}=1}^{N_{R}}$ into an estimate of the desired user's data sequence $\hat{s}^{m}[i]$. After transmit redundancy removal and FFT transformation, we obtain the multiantenna counterpart of (13):

$$
\tilde{\mathbf{Y}}_{n_{r}}[i]=\sum_{n_{t}=1}^{N_{T}} \tilde{\mathbf{H}}_{n_{r}, n_{t}} \cdot \tilde{\mathbf{X}}_{n_{t}}[i]+\tilde{\mathbf{Z}}_{n_{r}}[i]
$$

where $\tilde{\mathbf{Y}}_{n_{r}}[i]:=\left[\tilde{\mathbf{y}}_{n_{r}}[i N], \ldots, \tilde{\mathbf{y}}_{n_{r}}[(i+1) N-1]\right]$ stacks $N$ consecutive received chip blocks $\tilde{\mathbf{y}}_{n_{r}}[n]$ at the $n_{r}$ th receive antenna, $\tilde{\mathbf{H}}_{n_{r}, n_{t}}$ is the diagonal FD channel matrix from the $n_{t}$ th transmit to the $n_{r}$ th receive antenna, and $\tilde{\mathbf{X}}_{n_{t}}[i]$ and $\tilde{\mathbf{Z}}_{n_{r}}[i]$ are similarly defined as $\tilde{\mathbf{Y}}_{n_{r}}$ [i]. From (28) and (30), we can conclude that our transceiver retains the user orthogonality at each receive antenna, irrespective of the underlying frequency-selective multipath channels. As in the singleantenna case, a low-complexity block despreading operation with the desired user's composite code vector $\mathbf{c}^{m}[i]$ deterministically removes the MUI at each receive antenna:

$$
\overline{\mathbf{Y}}_{n_{r}}^{m}[i]:=\tilde{\mathbf{Y}}_{n_{r}}[i] \cdot \mathbf{c}^{m}[i]^{*}=\sum_{n_{t}=1}^{N_{T}} \tilde{\mathbf{H}}_{n_{r}, n_{t}} \cdot \overline{\mathbf{s}}_{n_{t}}^{m}[i]+\overline{\mathbf{z}}_{n_{r}}^{m}[i] .
$$

Hence, our transceiver successfully converts (through block despreading) a multiuser MIMO detection problem into an equivalent single-user MIMO equalization problem. 


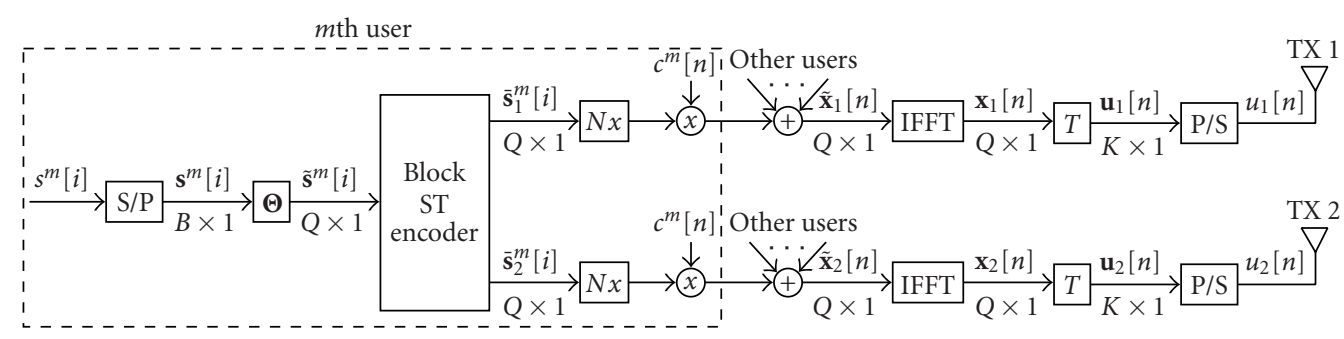

FIgURE 3: STBC MCBS-CDMA downlink transmission scheme.

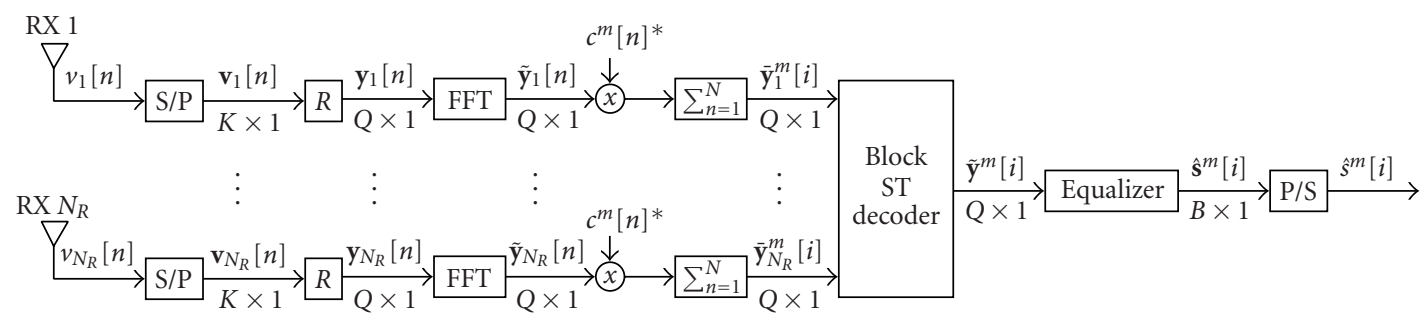

FIgURE 4: MUI-resilient STBC/MCBS-CDMA MIMO reception scheme.

\subsection{Single-user space-time decoding and equalization}

After MUI elimination, the information blocks $\mathbf{s}^{m}[i]$ still need to be decoded from the received block despread sequences $\left\{\overline{\mathbf{y}}_{n_{r}}^{m}[i]\right\}_{n_{r}=1}^{N_{R}}$. Our ST decoder decomposes into three steps: an initial ST decoding step, a transmit stream separation step for each receive antenna, and, finally, a receive antenna combining step.

The initial ST decoding step considers two consecutive symbol blocks $\left\{\overline{\mathbf{y}}_{n_{r}}^{m}[2 i]\right.$ and $\left.\overline{\mathbf{y}}_{n_{r}}^{m}[2 i+1]\right\}$, both satisfying the block input/output relationship of (31). By exploiting the ST code structure of (27) as in [36], we arrive at

$$
\overline{\mathbf{y}}_{n_{r}}^{m}[2 i]=\tilde{\mathbf{H}}_{n_{r}, 1} \cdot \overline{\mathbf{s}}_{1}^{m}[2 i]+\tilde{\mathbf{H}}_{n_{r}, 2} \cdot \overline{\mathbf{s}}_{2}^{m}[2 i]+\overline{\mathbf{z}}_{n_{r}}^{m}[2 i],
$$
$\overline{\mathbf{y}}_{n_{r}}^{m}[2 i+1]^{*}=-\tilde{\mathbf{H}}_{n_{r}, 1}^{*} \cdot \overline{\mathbf{s}}_{2}^{m}[2 i]+\tilde{\mathbf{H}}_{n_{r}, 2}^{*} \cdot \overline{\mathbf{s}}_{1}^{m}[2 i]+\overline{\mathbf{z}}_{n_{r}}^{m}[2 i+1]^{*}$.

Combining (32) and (33) into a single block matrix form, we obtain

$$
\begin{aligned}
& \underbrace{\left[\begin{array}{c}
\overline{\mathbf{y}}_{n_{r}}^{m}[2 i] \\
\overline{\mathbf{y}}_{n_{r}}^{m}[2 i+1]^{*}
\end{array}\right]}_{\tilde{\mathbf{r}}_{n_{r}}^{m}[i]} \\
& =\underbrace{\left[\begin{array}{cc}
\tilde{\mathbf{H}}_{n_{r}, 1} & \tilde{\mathbf{H}}_{n_{r}, 2} \\
\tilde{\mathbf{H}}_{n_{r}, 2}^{*} & -\tilde{\mathbf{H}}_{n_{r}, 1}^{*}
\end{array}\right]}_{\tilde{\mathbf{H}}_{n_{r}}} \cdot\left[\begin{array}{c}
\tilde{\mathbf{s}}^{m}[2 i] \\
\tilde{\mathbf{s}}^{m}[2 i+1]
\end{array}\right]+\underbrace{\left[\begin{array}{c}
\overline{\mathbf{z}}_{n_{r}}^{m}[2 i] \\
\overline{\mathbf{z}}_{n_{r}}^{m}[2 i+1]^{*}
\end{array}\right]}_{\tilde{\eta}_{n_{r}}^{m}[i]},
\end{aligned}
$$

where $\overline{\mathbf{s}}_{1}^{m}[2 i]=\widetilde{\mathbf{s}}^{m}[2 i]$ and $\overline{\mathbf{s}}_{2}^{m}[2 i]=\widetilde{\mathbf{s}}^{m}[2 i+1]$ follow from (27). From the structure of $\overline{\mathbf{H}}_{n_{r}}$ in (34), we can deduce that our transceiver retains the orthogonality among transmit streams at each receive antenna for each tone separately, regardless of the underlying frequency-selective multipath channels. A similar property was also encountered in the classical Alamouti scheme but only for single-user frequency-flat fading multipath channels [34].

The transmit stream separation step relies on this property to deterministically remove the transmit stream interference through low-complexity linear processing. We define the $Q \times Q$ matrix $\tilde{\mathbf{D}}_{n_{r}}$ with nonnegative diagonal entries as $\tilde{\mathbf{D}}_{n_{r}}:=\left[\tilde{\mathbf{H}}_{n_{r}, 1} \cdot \tilde{\mathbf{H}}_{n_{r}, 1}^{*}+\tilde{\mathbf{H}}_{n_{r}, 2} \cdot \tilde{\mathbf{H}}_{n_{r}, 2}^{*}\right]^{1 / 2}$. From (34), we can verify that the channel matrix $\overline{\mathbf{H}}_{n_{r}}$ satisfies $\overline{\mathbf{H}}_{n_{r}}^{H} \cdot \overline{\mathbf{H}}_{n_{r}}=\mathbf{I}_{2} \otimes \tilde{\mathbf{D}}_{n_{r}}^{2}$, where $\otimes$ stands for Kronecker product. Based on $\overline{\mathbf{H}}_{n_{r}}$ and $\tilde{\mathbf{D}}_{n_{r}}$, we can construct a unitary matrix $\overline{\mathbf{U}}_{n_{r}}:=\overline{\mathbf{H}}_{n_{r}} \cdot\left(\mathbf{I}_{2} \otimes \tilde{\mathbf{D}}_{n_{r}}^{-1}\right)$, which satisfies $\overline{\mathbf{U}}_{n_{r}}^{H} \cdot \overline{\mathbf{U}}_{n_{r}}=\mathbf{I}_{2 Q}$ and $\overline{\mathbf{U}}_{n_{r}}^{H} \cdot \overline{\mathbf{H}}_{n_{r}}=\mathbf{I}_{2} \otimes \tilde{\mathbf{D}}_{n_{r}}$. Performing unitary combining on (34) (through $\overline{\mathbf{U}}_{n_{r}}^{H}$ ) collects the transmit antenna diversity at the $n_{r}$ th receive antenna:

$$
\begin{aligned}
\underbrace{\left[\begin{array}{c}
\hat{\mathbf{y}}_{n_{r}}^{m}[2 i] \\
\dot{\mathbf{y}}_{n_{r}}^{m}[2 i+1]
\end{array}\right]}_{\hat{\mathbf{r}}_{n_{r}}^{m}[i]}: & =\overline{\mathbf{U}}_{n_{r}}^{H} \cdot \overline{\mathbf{r}}_{n_{r}}^{m}[i] \\
& =\left[\begin{array}{c}
\tilde{\mathbf{D}}_{n_{r}} \cdot \tilde{\mathbf{s}}^{m}[2 i] \\
\tilde{\mathbf{D}}_{n_{r}} \cdot \tilde{\mathbf{s}}^{m}[2 i+1]
\end{array}\right]+\underbrace{\left[\begin{array}{c}
\hat{\mathbf{z}}_{n_{r}}^{m}[2 i] \\
\hat{\mathbf{z}}_{n_{r}}^{m}[2 i+1]
\end{array}\right]}_{\hat{\boldsymbol{\eta}}_{n_{r}}^{m}[i]},
\end{aligned}
$$

where the resulting noise $\dot{\boldsymbol{\eta}}_{n_{r}}^{m}[i]:=\overline{\mathbf{U}}_{n_{r}}^{H} \cdot \overline{\boldsymbol{\eta}}_{n_{r}}^{m}[i]$ is still white with variance $\sigma_{w}^{2}$. Since multiplying with a unitary matrix preserves ML optimality, we can deduce from (35) that the symbol blocks $\tilde{\mathbf{s}}^{m}[2 i]$ and $\tilde{\mathbf{s}}^{m}[2 i+1]$ can be decoded separately in an optimal way. As a result, the different symbol blocks $\tilde{\mathbf{s}}^{m}[i]$ can be detected independently from

$$
\dot{\mathbf{y}}_{n_{r}}^{m}[i]=\tilde{\mathbf{D}}_{n_{r}} \cdot \tilde{\mathbf{s}}^{m}[i]+\dot{\mathbf{z}}_{n_{r}}^{m}[i] .
$$

Stacking the blocks from the different receive antennas $\left\{\hat{\mathbf{y}}_{n_{r}}^{m}[i]\right\}_{n_{r}=1}^{N_{R}}$ for the final receive antenna combining step, we 
obtain

$$
\underbrace{\left[\begin{array}{c}
\hat{\mathbf{y}}_{1}^{m}[i] \\
\vdots \\
\dot{\mathbf{y}}_{N_{R}}^{m}[i]
\end{array}\right]}_{\hat{\mathbf{y}}^{m}[i]}=\underbrace{\left[\begin{array}{c}
\tilde{\mathbf{D}}_{1} \\
\vdots \\
\tilde{\mathbf{D}}_{N_{R}}
\end{array}\right]}_{\mathbf{H}} \cdot \tilde{\mathbf{s}}^{m}[i]+\underbrace{\left[\begin{array}{c}
\dot{\mathbf{z}}_{1}^{m}[i] \\
\vdots \\
\dot{\mathbf{z}}_{N_{R}}^{m}[i]
\end{array}\right]}_{\dot{\mathbf{z}}^{m}[i]}
$$

At this point, we have only collected the transmit antenna diversity at each receive antenna, but still need to collect the receive antenna diversity. We define the $Q \times Q$ matrix $\tilde{\mathbf{D}}$ with nonnegative diagonal entries as $\tilde{\mathbf{D}}:=\left[\sum_{n_{t}=1}^{N_{T}} \sum_{n_{r}=1}^{N_{R}} \tilde{\mathbf{H}}_{n_{r}, n_{t}}\right.$. $\left.\tilde{\mathbf{H}}_{n_{r}, n_{t}}^{*}\right]^{1 / 2}$. From (37), we can verify that: $\mathbf{H}^{H} \cdot \mathbf{H}=\tilde{\mathbf{D}}^{2}$. Based on $\mathbf{H}$ and $\tilde{\mathbf{D}}$, we can construct a tall unitary matrix $\mathbf{U}:=\mathbf{H}$. $\tilde{\mathbf{D}}^{-1}$, which satisfies $\mathbf{U}^{H} \cdot \mathbf{U}=\mathbf{I}_{Q}$ and $\mathbf{U}^{H} \cdot \mathbf{H}=\tilde{\mathbf{D}}$. Gathering the receive antenna diversity through multiplying (37) with $\mathbf{U}^{H}$, we finally obtain

$$
\tilde{\mathbf{y}}^{m}[i]:=\mathbf{U}^{H} \cdot \dot{\mathbf{y}}^{m}[i]=\tilde{\mathbf{D}} \cdot \boldsymbol{\Theta} \cdot \mathbf{s}^{m}[i]+\tilde{\mathbf{z}}^{m}[i],
$$

where the resulting noise $\tilde{\mathbf{z}}^{m}[i]:=\mathbf{U}^{H} \cdot \dot{\mathbf{z}}^{m}[i]$ is still white with variance $\sigma_{w}^{2}$. Since the multiplication with a tall unitary matrix, which does not remove information, preserves ML decoding optimality, the blocks $\mathbf{s}^{m}[i]$ can be optimally decoded from (38). Furthermore, since (38) has the same structure as its single-antenna counterpart in (14), the design of the linear precoder $\Theta$ in Section 2.3 and the different equalization options that we have discussed in Section 3 can be applied here as well. Specifically, with $L_{t}$ the number of taps of the underlying multipath channels, the ML detector achieves the full diversity order of $N_{T} N_{R} L_{t}$, hence, both multi-antenna and multipath diversity. The transmit antenna diversity is enabled at the transmitter by the space-time encoder and collected at each receive antenna by the transmit stream separation step. The receive antenna diversity is collected by the final receive antenna combining step. The multipath diversity is enabled at the transmitter by the linear precoder, and extracted at the receiver by the ML joint equalization and decoding step.

\section{SIMULATION RESULTS}

We consider the downlink of an MCBS-CDMA system, operating at a carrier frequency of $F_{c}=2 \mathrm{GHz}$ and transmitting with a chip rate of $R_{c}=1 / T_{c}=4.096 \mathrm{MHz}$. Each user's bit sequence is QPSK modulated with $n_{b}=2$ bits per symbol. To assess the performance of the MCBS-CDMA system, we have selected ITU's outdoor-to-indoor and pedestrian B channel model, which models typical urban propagation environments. The main parameters of this tapped delay line model are summarized in Table 8 . Hence, the multipath channel has $L_{t}=6$ Rayleigh fading taps with a maximum excess delay of $\tau_{\max }=3700 \mathrm{~ns}$, resulting in a minimum channel order of $L_{\min }=\left\lceil\tau_{\max } / T_{c}\right\rceil=16$. To satisfy the IBI removal condition $L \geq L_{\text {min }}$, we choose the CP length $L=32$. This specific design can even handle a maximum excess delay of $T_{g}=L T_{c}=7812.5 \mathrm{~ns}$, with $T_{g}$ the guard time. However, a larger transmit redundancy can be used to handle more ICI.
TABLE 8: Parameters of the ITU pedestrian B channel.

\begin{tabular}{ccc}
\hline Tap & Excess delay $(\mathrm{ns})$ & Average relative power $(\mathrm{dB})$ \\
\hline 1 & 0 & 0 \\
2 & 200 & -0.9 \\
3 & 800 & -4.9 \\
4 & 1200 & -8.0 \\
5 & 2300 & -7.8 \\
6 & 3700 & -23.9 \\
\hline
\end{tabular}

TABle 9: Main MCBS-CDMA system parameters.

\begin{tabular}{ll} 
Carrier frequency & $F_{c}=2 \mathrm{GHz}$ \\
Chip rate & $R_{c}=4.096 \mathrm{MHz}$ \\
Modulation format & $n_{b}=2(\mathrm{QPSK})$ \\
Initial block length & $B=224$ \\
Cyclic prefix length & $L=32$ \\
Number of subcarriers & $Q=256$ \\
Transmitted block length & $K=288$ \\
Symbol rate & $R_{s}=199 \mathrm{kHz}$ \\
\hline
\end{tabular}

Adversely, a smaller transmit redundancy is allowed if less ICI has to be handled. To limit the overhead, we choose the number of subcarriers $Q=8 L=256$, leading to a transmitted block length $K=Q+L=288$. Hence, the information symbols are parsed into blocks of $B=Q-L=224$ symbols and linearly precoded into blocks of size $Q=256$. The $Q \times B$ precoding matrix, $\Theta$, constitutes the first $B$ columns of the $Q \times Q$ DCT matrix [22]. The precoded symbol blocks are subsequently block spread by a real orthogonal Walsh-Hadamard spreading code of length $N=16$, along with a complex random scrambling code. For the above parameters, this results in a channel symbol rate of $R_{s}=(B / K)(1 / N) R_{c}=199 \mathrm{kHz}$. For convenience, the main MCBS-CDMA system parameters are summarized in Table 9.

In the following, we show the average bit error rate (BER) versus the average received SNR for three different test cases. Here, the SNR is defined as the average received energy per bit of the desired user versus the noise power spectral density. Section 5.1 compares the different single-user equalization options, from a BER performance as well as a complexity point of view. Section 5.2 compares the BER performance of the proposed MCBS-CDMA transceiver with two competing CDMA transceivers. Finally, Section 5.3 discusses the BER performance of the SIBC-MCBS-CDMA transceiver in different propagation environments.

\subsection{Comparison of different equalization options}

We test the different equalization options, discussed in Section 3, for a fully-loaded MCBS-CDMA system with $M=$ 16 active users.

Figure 5 compares the performance of the different block linear equalizers (BLEs) and BDFEs that perform joint equalization and decoding. As a reference also, the performance of a system without linear precoding (uncoded) as well as the optimal ML performance are shown. Clearly, the system without linear precoding only achieves diversity 1 , whereas 


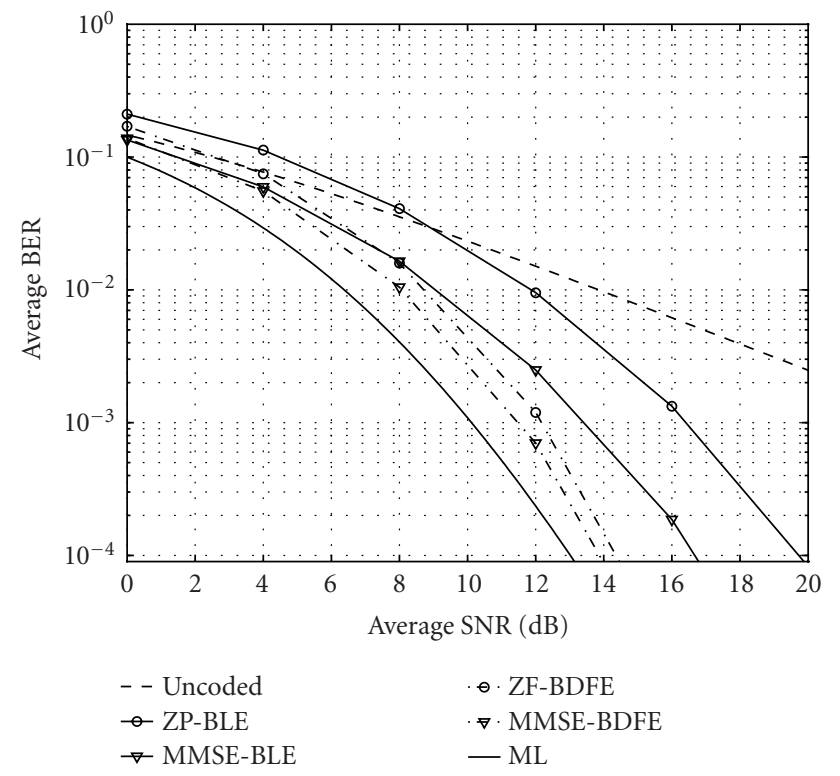

FIgURe 5: Performance comparison of joint block linear equalization (BLE) and decoding versus joint block decision feedback equalization (BDFE) and decoding for fully-loaded MCBS-CDMA system with $M=16$ users. Both ZF and MMSE critera are considered. Uncoded and ML performances are shown as a reference.

ML detection achieves the full frequency-diversity gain $L_{t}=$ 6 . The ZF-BLE performs worse than the uncoded system at low SNR but better at high SNR (SNR $\geq 9 \mathrm{~dB})$. The MMSEBLE always outperforms the uncoded system and achieves a diversity gain between 1 and $L_{t}=6$. At a BER of $10^{-3}$, it realizes a $3 \mathrm{~dB}$ gain compared to its $\mathrm{ZF}$ counterpart. The nonlinear ZF- and MMSE-BDFEs outperform their respective linear counterparts, although this effect is more pronounced for the ZF than for the MMSE criterion. For a target BER of $10^{-3}$, the MMSE-BDFE exhibits a $1.9 \mathrm{~dB}$ gain relative to the MMSE-BLE, whereas the ZF-BDFE exhibits a $4.2 \mathrm{~dB}$ gain relative to the ZF-BLE. Furthermore, the MMSE-BDFE marginally outperforms the ZF-BDFE by $0.7 \mathrm{~dB}$, and comes within $1.4 \mathrm{~dB}$ of the optimal ML detector.

Figure 6 compares the performance of separate PT-LE and decoding versus joint block linear equalization (BLE) and decoding, both of which perform linear equalization. On the one hand, the ZF-PT-LE always performs worse than the uncoded system, due to the excessive noise enhancement caused by the presence of channel nulls. For a target BER of $10^{-2}$, the ZF-BLE outperforms its corresponding ZF-PT-LE by $7.4 \mathrm{~dB}$. On the other hand, the MMSE-PT-LE performs within $0.3 \mathrm{~dB}$ of its corresponding MMSE-BLE, and, thus, achieves a diversity gain between 1 and $L_{t}=6$. The MMSEBLE, on its turn, outperforms the uncoded system by $4.8 \mathrm{~dB}$ and comes within $2.7 \mathrm{~dB}$ of the optimal ML detector.

Tables 10 and 11 summarize the complexity results for the different MCBS-CDMA equalization options. Table 10 compares the initialization complexity of the different equalization options. The initialization complexity of the ZF-BLE, which is similar to that of the ZF-BDFE, involves an opera-

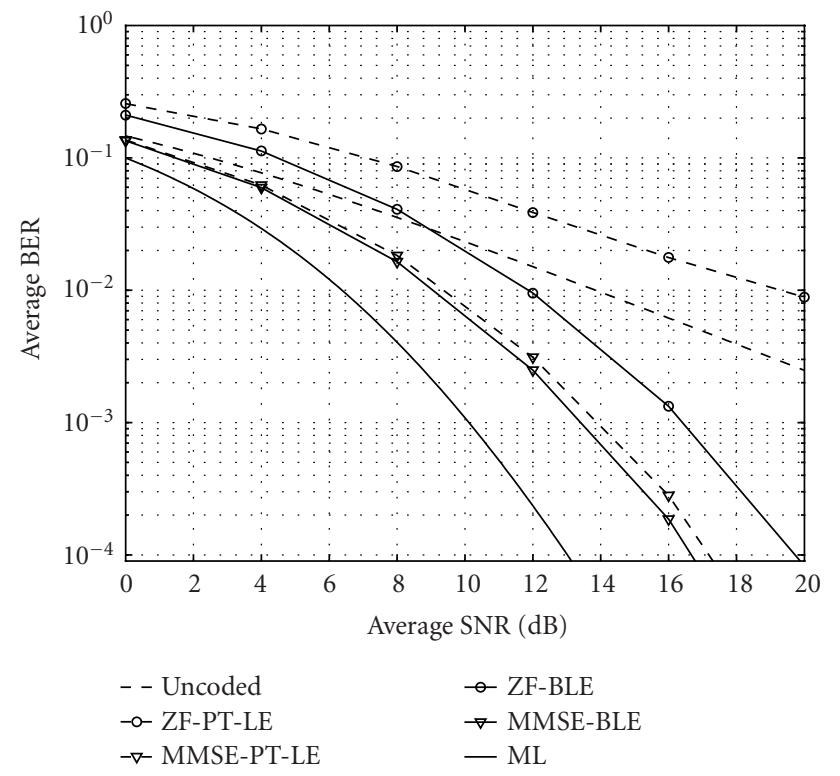

Figure 6: Performance comparison of separate PT-LE and decoding versus joint block linear equalization (BLE) and decoding for a fully-loaded MCBS-CDMA system with $M=16$ users. Both the ZF and the MMSE criteria are considered. Uncoded and ML performances are shown as a reference.

TABLE 10: Comparison of the initialization complexity of the different MCBS-CDMA equalization options.

\begin{tabular}{lccc}
\hline & \multicolumn{3}{c}{$\begin{array}{c}\text { Initialization } \\
\text { adds }\end{array}$} \\
\hline ML & - & - & dts \\
ZF-BLE & $998 \mathrm{M}$ & $998 \mathrm{M}$ & $6.0 \mathrm{G}$ \\
MMSE-BLE & $512 \mathrm{M}$ & $32 \mathrm{M}$ & $1.6 \mathrm{G}$ \\
ZF-BDFE & $1.0 \mathrm{G}$ & $1.0 \mathrm{G}$ & $6.1 \mathrm{G}$ \\
MMSE-BDFE & $527 \mathrm{M}$ & $47 \mathrm{M}$ & $1.7 \mathrm{G}$ \\
ZF-PT-LE & $0.5 \mathrm{k}$ & - & $1.5 \mathrm{k}$ \\
MMSE-PT-LE & $0.5 \mathrm{k}$ & $0.3 \mathrm{k}$ & $2.3 \mathrm{k}$ \\
\hline
\end{tabular}

TABLE 11: Comparison of the data processing complexity of the different MCBS-CDMA equalization options.

\begin{tabular}{lccc}
\hline & \multicolumn{3}{c}{$\begin{array}{c}\text { Data processing } \\
\text { adds/s }\end{array}$} \\
\hline ML & $1.7 \cdot 10^{131} \mathrm{G}$ & $3.9 \cdot 10^{131} \mathrm{G}$ & $1.5 \cdot 10^{132} \mathrm{G}$ \\
ZF-BLE & $51 \mathrm{M}$ & $51 \mathrm{M}$ & $305 \mathrm{M}$ \\
MMSE-BLE & $51 \mathrm{M}$ & $51 \mathrm{M}$ & $305 \mathrm{M}$ \\
ZF-BDFE & $96 \mathrm{M}$ & $95 \mathrm{M}$ & $573 \mathrm{M}$ \\
MMSE-BDFE & $96 \mathrm{M}$ & $95 \mathrm{M}$ & $573 \mathrm{M}$ \\
ZF-PT-LE & $1 \mathrm{M}$ & $2 \mathrm{M}$ & $9 \mathrm{M}$ \\
MMSE-PT-LE & $1 \mathrm{M}$ & $2 \mathrm{M}$ & $9 \mathrm{M}$ \\
\hline
\end{tabular}

tion count of 998 Mmpys and 998 Madds, and a data transfer count of $6.0 \mathrm{Gdts}$. The initialization complexity of the MMSE-BLE, which is similar to that of the MMSE-BDFE, involves 2 times less multiplications, 30 times less additions, 
and 3.7 times less data transfers. Specifically, it amounts to an operation count of 512 Mmpys and 32 Madds, and a data transfer count of $1.6 \mathrm{Gdts}$. On the other hand, the MMSEPT-LE involves an initialization complexity, which is between 5 and 6 orders of magnitude smaller than that of its corresponding MMSE-BLE. Specifically, its initialization complexity amounts to an operation count of $0.5 \mathrm{kmpys}$ and 0.3 kadds, and a data transfer count of $2.3 \mathrm{kdts}$.

Table 11 compares the data processing complexity of the different equalization options. Note that the equalizer block operates at a rate which is $B$ times lower than the symbol rate $R_{s}$, that is, $R_{\mathrm{eq}}=R_{s} / B=889 \mathrm{~Hz}$. The data processing complexity of the optimal ML algorithm is astronomically high, which certainly prohibits implementation, even on the most advanced quantum computers. The BLEs have a data processing complexity, which amounts to an operation count of $51 \mathrm{Mmpys} / \mathrm{s}$ and $51 \mathrm{Madds} / \mathrm{s}$, and a data transfer bandwidth of $305 \mathrm{Mdts} / \mathrm{s}$. On the one hand, the data processing complexity of the BDFEs is approximately twice that of the BLEs. On the other hand, the data processing complexity of the PTLEs is roughly between 1 and 2 orders of magnitude lower than that of the corresponding BLEs. Specifically, it amounts to an operation count of $1 \mathrm{Mmpys} / \mathrm{s}$ and $2 \mathrm{Madds} / \mathrm{s}$, and a data transfer bandwidth of $9 \mathrm{Mdts} / \mathrm{s}$.

\subsection{Comparison of different CDMA transceivers}

In the following, we compare three different CDMA transceivers.

(1) The first transceiver applies the downlink DS-CDMA transmission scheme used in $3 \mathrm{G}$ cellular standards, performing classical symbol spreading. The receiver employs either a classical RAKE combiner or an MMSE time-domain chip equalizer (TD-CE) $[3,4,5$, $6,7,8]$ based on perfect CSI. The number of fingers in the RAKE combiner equals $L_{t}=6$, while the order of the chip equalizer equals $Q_{c}=23$. The bandwidth efficiency of this first transceiver, supporting $M_{1}$ users, can be calculated as $\epsilon_{1}=M_{1} / N$, where $N$ is the length of the Walsh-Hadamard spreading codes.

(2) The second transceiver applies the downlink MCCDMA transmission scheme, performing classical symbol spreading followed by OFDM modulation [14, $15,16]$. The receiver employs an MMSE frequencydomain chip equalizer (FD-CE) based on perfect CSI. The bandwidth efficiency of this second transceiver, supporting $M_{2}$ users, can be calculated as $\epsilon_{2}=$ $\epsilon_{\mathrm{MC}-\mathrm{CDMA}}=M_{2} B_{2} /\left(B_{2} N+L\right)$, where $B_{2}$ is the initial block length and $Q_{2}=B_{2} N$ is the number of subcarriers. The overhead of $L$ stems from the CP for IBI removal.

(3) The third transceiver is our MCBS-CDMA transceiver that we have derived in Section 2, combining blockspread CDMA and linearly-precoded OFDM. The receiver employs an MMSE PT-LE or ML detection. As discussed in Section 2.1, the bandwidth efficiency of this third transceiver, supporting $M_{3}$ users, can be calculated as $\epsilon_{3}=\epsilon_{\mathrm{MCBS}-\mathrm{CDMA}}=M_{3} B_{3} / N\left(B_{3}+2 L\right)$, where

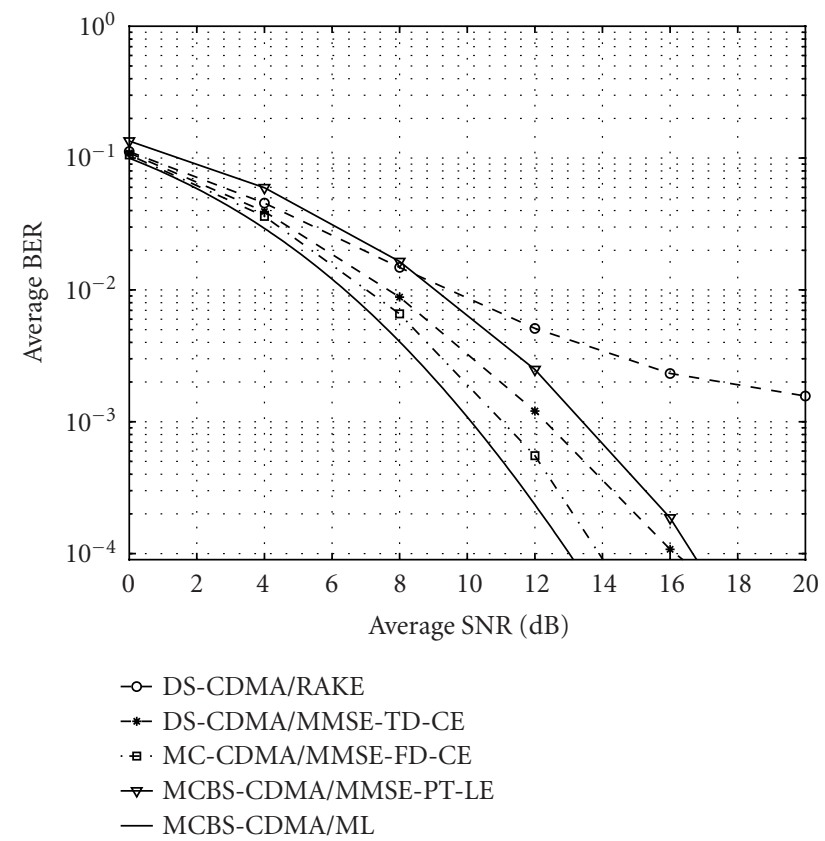

Figure 7: Comparison of DS-CDMA, MC-CDMA, and MCBSCDMA for small system load with $M_{1}=3, M_{2}=3$, and $M_{3}=$ 4 users, respectively: RAKE and MMSE-TD-CE for DS-CDMA; MMSE-FD-CE for MC-CDMA; MMSE-PT-LE and ML for MCBSCDMA.

$B_{3}$ is the initial block length and $Q_{3}=B_{3}+L$ is the number of tones. The overhead of $2 L$ stems from the redundant linear precoding, on the one hand, and the $\mathrm{CP}$, on the other.

In order to make a fair comparison between the three transceivers, we should force their respective bandwidth efficiencies to be the same, that is, $\epsilon_{1}=\epsilon_{2}=\epsilon_{3}$. This leads to the following relationship between the number of users to be supported by the different transceivers: $M_{2}=\left(\left(B_{2} N+\right.\right.$ $\left.L) / B_{2} N\right) M_{1}$, and $M_{3}=\left(\left(B_{3}+2 L\right) / B_{3}\right) M_{1}$. With $N=16$, $L=32, Q_{2}=Q_{3}=8 L=256, B_{2}=16$, and $B_{3}=224$, we can derive that $M_{2}=(9 / 8) M_{1}$ and $M_{3}=(9 / 7) M_{1}$. Furthermore, we ensure that the total transmit power is the same for the different transceivers.

Figure 7 compares the performance of the different transceivers for a small system load with $M_{1}=3, M_{2}=3$, and $M_{3}=4$ users, respectively $\left(\epsilon_{1} \approx \epsilon_{2} \approx \epsilon_{3}\right)$. The DSCDMA RAKE receiver starts flooring off at $10^{-3}$, due to ISI/ICI and associated MUI. The DS-CDMA MMSE-TD$\mathrm{CE}$ actively suppresses these interferences and achieves a significant performance improvement compared to the RAKE. On the other hand, the MC-CDMA MMSE-FD-CE has the same performance as the DS-CDMA MMSE-TD-CE at low SNR (SNR $<8$ ), but clearly outperforms it at high SNR. Furthermore, the MCBS-CDMA MMSE-PT-LE that deterministically removes the MUI but still suffers from ISI performs worse than both the DS-CDMA MMSE-TD-CE and the MC-CDMA MMSE-FD-CE. Specifically, for a target BER of $10^{-4}$, the DS-CDMA MMSE-TD-CE realizes a $0.5 \mathrm{~dB}$ gain 


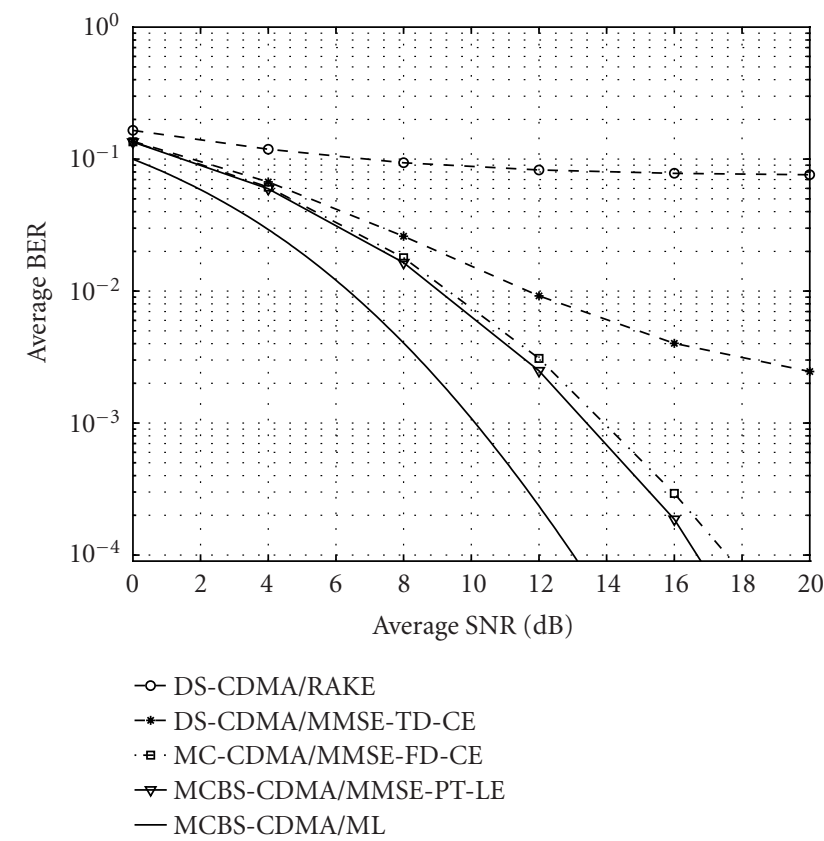

FIgure 8: Comparison of DS-CDMA, MC-CDMA, and MCBSCDMA for large system load with $M_{1}=12, M_{2}=14$, and $M_{3}=$ 16 users, respectively: RAKE and MMSE-TD-CE for DS-CDMA; MMSE-FD-CE for MC-CDMA; MMSE-PT-LE and ML for MCBSCDMA.

compared to the MCBS-CDMA MMSE-PT-LE, whereas the MC-CDMA MMSE-FD-CE realizes a $2.8 \mathrm{~dB}$ gain. Finally, the optimal MCBS-CDMA ML achieves the full diversity gain of $L_{t}=6$.

Figure 8 depicts the performance of the different transceivers for a large system load with $M_{1}=12, M_{2}=14$, and $M_{3}=16$ users, respectively $\left(\epsilon_{1} \approx \epsilon_{2} \approx \epsilon_{3}\right)$. The DSCDMA RAKE receiver clearly suffers from a BER floor at $8 \cdot 10^{-2}$, since it does not cope at all with the increased MUI. Although the DS-CDMA MMSE-TD-CE still outperforms the RAKE, its performance also starts flooring off, because it does not completely suppress these interferences at high SNR. Indeed, the existence of a ZF solution for DSCDMA TD chip equalization requires multichannel reception at the MS [7, 8]. Hence, both DS-CDMA receivers suffer from a BER saturation level that increases with the system load $M_{1}$. Likewise, since the MC-CDMA MMSE-FDCE does not deterministically suppress the MUI either, its performance is also affected by the increased MUI. However, unlike the DS-CDMA MMSE-TD-CE, it does not suffer from a BER floor, since it more effectively copes with the ICI through CP. In contrast with DS-CDMA and MC-CDMA, MCBS-CDMA is an MUI-free CDMA transceiver, such that its performance remains unaffected by the increased MUI. Consequently, even at large system load, the MCBS-CDMA MMSE-PT-LE achieves a diversity order between 1 and $L_{t}=$ 6. Furthermore, the MCBS-CDMA MMSE-PT-LE now performs better than both the DS-CDMA MMSE-TD-CE and the MC-CDMA MMSE-FD-CE. Specifically, for a target BER

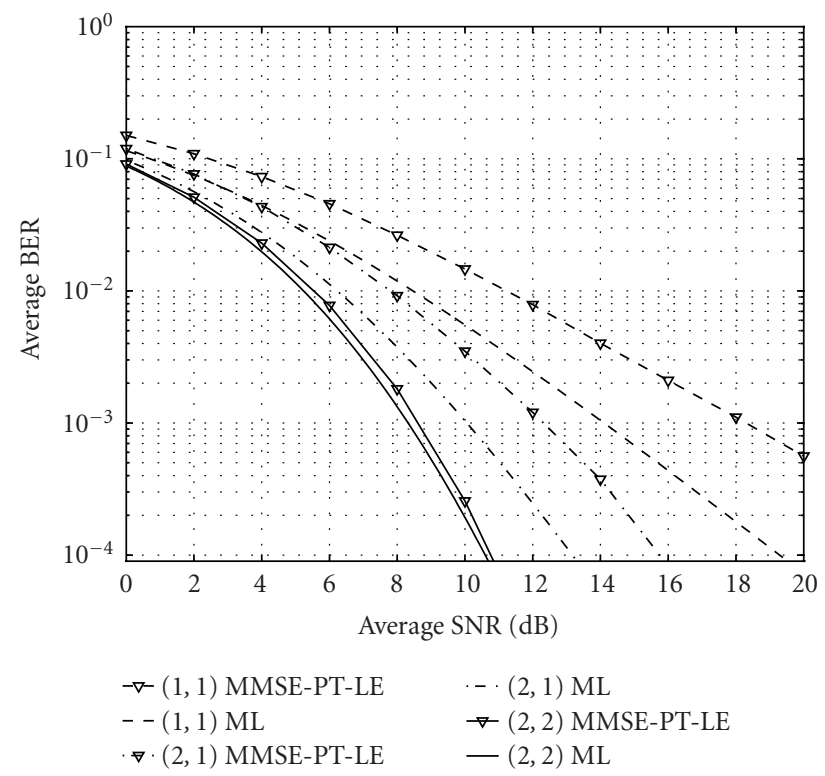

Figure 9: Performance of STBC-MCBS-CDMA for channels with small delay spread. Different MIMO system setups, ranging from $(1,1)$ over $(2,1)$ to $(2,2)$. MMSE-PT-LE and ML detection.

of $3 \cdot 10^{-3}$, the MCBS-CDMA MMSE-PT-LE outperforms the DS-CDMA MMSE-TD-CE by $6.8 \mathrm{~dB}$. Additionally, for a target BER of $10^{-4}$, the MCBS-CDMA MMSE-PT-LE performs $1 \mathrm{~dB}$ better than the MC-CDMA MMSE-FD-CE. Finally, the optimal MCBS-CDMA ML still achieves the full diversity gain of $L_{t}=6$.

\subsection{Performance of space-time block-coded MCBS-CDMA}

We test our STBC-MCBS-CDMA transceiver of Section 4, employing a cascade of STBC and MCBS-CDMA, for three different MIMO system setups $\left(N_{T}, N_{R}\right)$ : the $(1,1)$ setup, the $(2,1)$ setup with TX diversity only, and the $(2,2)$ setup with both TX and RX diversity. The system is fully loaded supporting $M=16$ active users. For each setup, the receiver employs an MMSE-PT-LE or an ML detector based on perfect CSI.

Figure 9 depicts the performance over a propagation environment with a small delay spread. The underlying multipath channel has $L_{t}=2$ chip-spaced Rayleigh fading taps of equal average power. For a target BER at $10^{-3}$, and focusing on the MMSE-PT-LE, the $(2,1)$ setup outperforms the $(1,1)$ setup by $6 \mathrm{~dB}$. The $(2,2)$ setup achieves, on its turn, a $3.7 \mathrm{~dB}$ gain compared to the $(2,1)$ setup. Comparing the MMSEPT-LE with its corresponding ML detector, it incurs a $4.2 \mathrm{~dB}$ loss for the $(1,1)$ setup, but only a $0.3 \mathrm{~dB}$ loss for the $(2,2)$ setup. So, the larger the number of transmit and/or receive antennas, the better the linear MMSE-PT-LE succeeds in extracting the full diversity of order $N_{T} N_{R} L_{t}$.

Figure 10 shows the performance over a propagation environment with a large delay spread. The underlying 


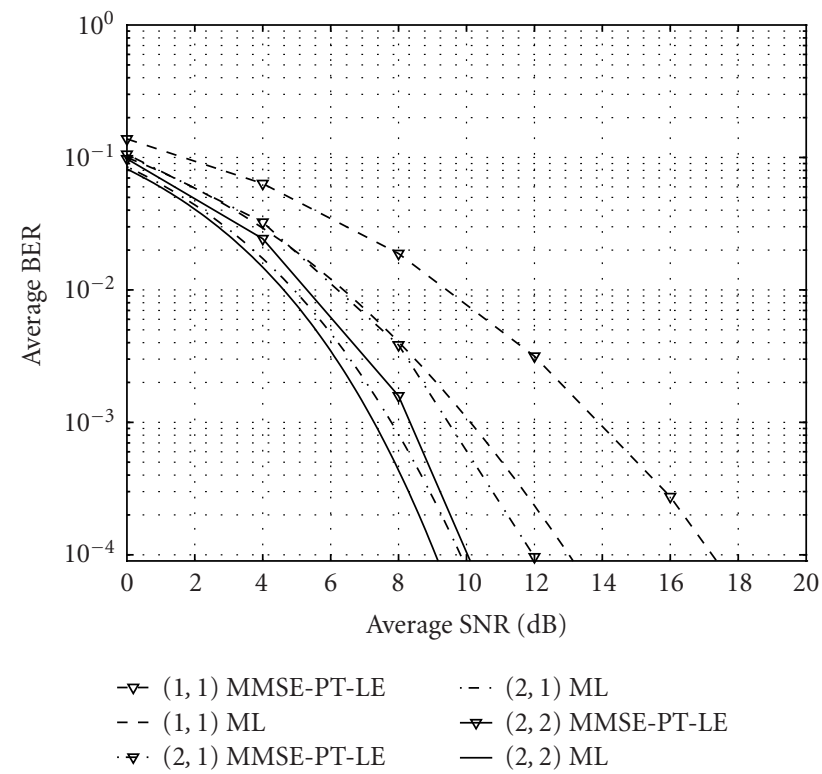

FIgUre 10: Performance of STBC-MCBS-CDMA for channels with large delay spread. Different MIMO system setups, ranging from $(1,1)$ over $(2,1)$ to $(2,2)$. MMSE-PT-LE and ML detection.

multipath channel, which is the ITU pedestrian B channel that we have introduced before, has $L_{t}=6$ Rayleigh fading taps. For a target BER at $10^{-3}$, and focusing on the MMSE-PT-LE, the $(2,1)$ setup outperforms the $(1,1)$ setup by $4.4 \mathrm{~dB}$, whereas the $(2,2)$ setup achieves, on its turn, a $1.1 \mathrm{~dB}$ gain compared to the $(2,1)$ setup. So, compared to Figure 9, the corresponding gains due to multiantenna diversity are now smaller because of the inherently larger underlying multipath diversity. Comparing the MMSE-PT-LE with its corresponding ML detector, it incurs a $0.9 \mathrm{~dB}$ loss for the $(2,2)$ setup.

\section{CONCLUSION}

To cope with the challenges of broadband cellular downlink communications, we have designed a novel multicarrier CDMA transceiver that enables significant performance improvements compared to $3 \mathrm{G}$ cellular systems, yielding gains of up to $6.8 \mathrm{~dB}$ in full load situations. To this end, our MCBS-CDMA transmission technique capitalizes on redundant block spreading and linear precoding to preserve the orthogonality among users and to enable full multipath diversity gains, regardless of the underlying multipath channels. The corresponding receiver relies on low-complexity block despreading to convert a difficult multiuser detection problem into an equivalent but simpler single-user equalization problem, for which any single-user equalizer allows to trade-off performance versus complexity. In this perspective, we have evaluated the performance and complexity of four different single-user equalization options for a realistic MCBS-CDMA cellular system that fits the UMTS channel bandwidth. On the one hand, the performance results show that, for a target BER of $10^{-3}$, the MMSE-BDFE exhibits a $1.9 \mathrm{~dB}$ gain relative to the MMSE-BLE, and comes within $1.4 \mathrm{~dB}$ of the optimal ML detector. Furthermore, the MMSE-PT-LE performs within $0.3 \mathrm{~dB}$ of the MMSE-BLE, while it is $3.6 \mathrm{~dB}$ away from the ML detector. On the other hand, the complexity estimates show that the initialization complexity of the MMSE-BDFE is similar to that of the MMSE-BLE, while its data processing complexity is approximately two times higher. Furthermore, the MMSE-PT-LE involves an initialization complexity, which is between 5 and 6 orders of magnitude smaller than that of the MMSEBLE, while its data processing complexity is roughly between 1 and 2 orders of magnitude smaller. Based on this study, we can conclude that the MMSE-PT-LE offers a good trade-off between performance and complexity. Finally, to increase the spectral efficiency and to improve the link reliability of multiple users in a broadband cellular network, we have demonstrated the rewarding synergy between MCBSCDMA and existing and evolving MIMO communication techniques. Specifically, our STBC-MCBS-CDMA transmission technique not only retains the orthogonality among users but also among the different transmit streams of each user. At the receiver, these properties, respectively, allow for deterministic ML user separation through low-complexity block despreading as well as deterministic transmit stream separation through simple linear processing. Consequently, ML equalization per transmit stream and per user achieves maximum multiantenna and multipath diversity gains for every user in the system, irrespective of the system load. Furthermore, the low-complexity MMSE-PT-LE approaches the optimal ML performance (within $0.9 \mathrm{~dB}$ for a $(2,2)$ system), and comes close to extracting the full diversity in reduced as well as full load settings.

\section{REFERENCES}

[1] H. Holma and A. Toskala, WCDMA for UMTS: Radio Access for Third Generation Mobile Communications, John Wiley \& Sons, New York, NY, USA, 2001.

[2] L. B. Milstein, "Wideband code division multiple access," IEEE Journal on Selected Areas in Communications, vol. 18, no. 8, pp. 1344-1354, 2000.

[3] A. Klein, "Data detection algorithms specially designed for the downlink of CDMA mobile radio systems," in IEEE 47th Vehicular Technology Conference (VTC '97), vol. 1, pp. 203207, Phoenix, Ariz, USA, May 1997.

[4] I. Ghauri and D. T. M. Slock, "Linear receivers for the DSCDMA downlink exploiting orthogonality of spreading sequences," in Proc. IEEE 32nd Asilomar Conference on Signals, Systems \& Computers, vol. 1, pp. 650-654, Pacific Grove, Calif, USA, November 1998.

[5] C. D. Frank, E. Visotsky, and U. Madhow, "Adaptive interference suppression for the downlink of a direct sequence CDMA system with long spreading sequences," The Journal of VLSI Signal Processing, vol. 30, no. 1-3, pp. 273-291, 2002.

[6] K. Hooli, M. Juntti, M. J. Heikkilä, P. Komulainen, M. Latvaaho, and J. Lilleberg, "Chip-level channel equalization in WCDMA downlink," EURASIP Journal on Applied Signal Processing, vol. 2002, no. 8, pp. 757-770, 2002.

[7] T. P. Krauss, W. J. Hillery, and M. D. Zoltowski, "Downlink specific linear equalization for frequency selective CDMA cellular systems," The Journal of VLSI Signal Processing, vol. 30, no. 1-3, pp. 143-161, 2002. 
[8] F. Petré, G. Leus, L. Deneire, M. Engels, M. Moonen, and H. De Man, "Adaptive chip equalization for DS-CDMA downlink with receive diversity," IEEE Transactions on Wireless Communications, May 2003, accepted subject to major revisions.

[9] L. J. Cimini Jr., "Analysis and simulation of a digital mobile channel using orthogonal frequency division multiplexing," IEEE Trans. Communications, vol. 33, no. 7, pp. 665-675, 1985.

[10] J. A. C. Bingham, "Multicarrier modulation for data transmission: an idea whose time has come," IEEE Communications Magazine, vol. 28, no. 5, pp. 5-14, 1990.

[11] Z. Wang and G. B. Giannakis, "Wireless multicarrier communications," IEEE Signal Processing Magazine, vol. 17, no. 3, pp. 29-48, 2000.

[12] W. Y. Zou and Y. Wu, "COFDM: an overview," IEEE Transactions on Broadcasting, vol. 41, no. 1, pp. 1-8, 1995.

[13] S. Hara and R. Prasad, "Overview of multicarrier CDMA," IEEE Communications Magazine, vol. 35, no. 12, pp. 126-133, 1997.

[14] N. Yee, J.-P. Linnartz, and G. Fettweis, "Multi-carrier CDMA in indoor wireless radio networks," in Proc. IEEE International Symposium on Personal, Indoor, and Mobile Radio Communications (PIMRC '93), vol. 1, pp. 109-113, Yokohama, Japan, September 1993.

[15] K. Fazel, S. Kaiser, and M. Schnell, "A flexible and high performance cellular mobile communications system based on orthogonal multi-carrier SSMA," Wireless Personal Communications, vol. 2, no. 1/2, pp. 121-144, 1995.

[16] S. Kaiser, "OFDM code-division multiplexing in fading channels," IEEE Trans. Communications, vol. 50, no. 8, pp. 1266 1273, 2002.

[17] V. M. DaSilva and E. S. Sousa, "Multicarrier orthogonal CDMA signals for quasi-synchronous communication systems," IEEE Journal on Selected Areas in Communications, vol. 12, no. 5, pp. 842-852, 1994.

[18] S. Kondo and L. B. Milstein, "Performance of multicarrier DS CDMA systems," IEEE Trans. Communications, vol. 44, no. 2, pp. 238-246, 1996.

[19] L. Vandendorpe, "Multitone spread spectrum multiple access communications system in a multipath Rician fading channel," IEEE Trans. Vehicular Technology, vol. 44, no. 2, pp. 327 337, 1995.

[20] G. B. Giannakis, Z. Wang, A. Scaglione, and S. Barbarossa, "AMOUR-generalized multicarrier transceivers for blind CDMA regardless of multipath," IEEE Trans. Communications, vol. 48, no. 12, pp. 2064-2076, 2000.

[21] Z. Wang and G. B. Giannakis, "Linearly precoded or coded OFDM against wireless channel fades?," in Proc. IEEE $3 \mathrm{rd}$ Workshop on Signal Processing Advances in Wireless Communications (SPAWC '01), pp. 267-270, Taiwan, China, March 2001.

[22] Z. Wang and G. B. Giannakis, "Complex-field coding for OFDM over fading wireless channels," IEEE Transactions on Information Theory, vol. 49, no. 3, pp. 707-720, 2003.

[23] A. Peled and A. Ruiz, "Frequency domain data transmission using reduced computational complexity algorithms," in Proc. IEEE Int. Conf. Acoustics, Speech, Signal Processing (ICASSP '80), vol. 5, pp. 964-967, Denver, Colo, USA, April 1980.

[24] G. H. Golub and C. F. Van Loan, Matrix Computations, Johns Hopkins University Press, Baltimore, Md, USA, 1996.

[25] A. Klein and P. W. Baier, "Linear unbiased data estimation in mobile radio systems applying CDMA," IEEE Journal on
Selected Areas in Communications, vol. 11, no. 7, pp. 10581066, 1993.

[26] A. Klein, G. K. Kaleh, and P. W. Baier, "Zero forcing and minimum mean-square-error equalization for multiuser detection in code-division multiple-access channels," IEEE Trans. Vehicular Technology, vol. 45, no. 2, pp. 276-287, 1996.

[27] A. Stamoulis, G. B. Giannakis, and A. Scaglione, "Block FIR decision-feedback equalizers for filterbank precoded transmissions with blind channel estimation capabilities," IEEE Trans. Communications, vol. 49, no. 1, pp. 69-83, 2001.

[28] G. J. Foschini and M. J. Gans, "On limits of wireless communications in a fading environment when using multiple antennas," Wireless Personal Communications, vol. 6, no. 3, pp. 311-335, 1998.

[29] G. G. Raleigh and J. M. Cioffi, "Spatio-temporal coding for wireless communication," IEEE Trans. Communications, vol. 46, no. 3, pp. 357-366, 1998.

[30] D. Gesbert, H. Bolcskei, D. Gore, and A. Paulraj, "MIMO wireless channels: capacity and performance prediction," in Proc. IEEE Global Telecommunications Conference (GLOBECOM '00), vol. 2, pp. 1083-1088, San Francisco, Calif, USA, November-December 2000.

[31] G. J. Foschini, "Layered space-time architecture for wireless communication in a fading environment when using multiple antennas," Bell Labs Technical Journal, vol. 1, no. 2, pp. 41-59, 1996.

[32] A. Paulraj and T. Kailath, "Increasing capacity in wireless broadcast systems using distributed transmission/directional reception (DTDR)," US Patent 5345599, Stanford University, Stanford, Calif, USA, September 1994.

[33] V. Tarokh, N. Seshadri, and A. R. Calderbank, "Space-time codes for high data rate wireless communication: performance criterion and code construction," IEEE Transactions on Information Theory, vol. 44, no. 2, pp. 744-765, 1998.

[34] S. M. Alamouti, "A simple transmit diversity technique for wireless communications," IEEE Journal on Selected Areas in Communications, vol. 16, no. 8, pp. 1451-1458, 1998.

[35] V. Tarokh, H. Jafarkhani, and A. R. Calderbank, "Space-time block codes from orthogonal designs," IEEE Transactions on Information Theory, vol. 45, no. 5, pp. 1456-1467, 1999.

[36] Z. Liu, G. B. Giannakis, B. Muquet, and S. Zhou, "Spacetime coding for broadband wireless communications," Wireless Communications and Mobile Computing, vol. 1, no. 1, pp. 35-53, 2001.

Frederik Petré was born in Tienen, Belgium, on December 12, 1974. He received the Electrical Engineering degree and the Ph.D. in applied sciences from the Katholieke Universiteit Leuven (KULeuven), Leuven, Belgium, in July 1997 and December 2003, respectively. In September 1997, he joined the Design Technology for Integrated Information and Communication Systems (DESICS) Division at the In-

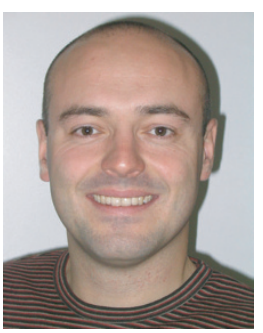
teruniversity Micro-Electronics Center (IMEC) in Leuven, Belgium. Within the Digital Broadband Terminals (DBATE) Group of DESICS, he first performed predoctoral research on wireline transceiver design for twisted pair, coaxial cable, and powerline communications. During the fall of 1998, he visited the Information Systems Laboratory (ISL) at Stanford University, California, USA, working on OFDM-based powerline communications. In January 1999, he joined the Wireless Systems (WISE) group of DESICS as a Ph.D. researcher, funded by the Institute for 
Scientific and Technological Research in Flanders (IWT). Since January 2004, he is a Senior Scientist within the Wireless Research group of DESICS. He is investigating the baseband signal processing algorithms and architectures for future wireless communication systems, like third generation $(3 \mathrm{G})$ and fourth generation (4G) cellular networks, and wireless local area networks (WLANs). His main research interests are modulation theory, multiple access schemes, channel estimation and equalization, and smart antenna and MIMO techniques. He is a Member of the ProRISC technical program committee and the IEEE Benelux Section on Communications and Vehicular Technology (CVT). He is a Member of the Executive Board and Project Leader of the Reconfigurable Radio Project of the Network of Excellence in Wireless Communications (NEWCOM), established under the sixth framework of the European Commission.

Geert Leus was born in Leuven, Belgium, in 1973. He received the Electrical Engineering degree and the Ph.D. degree in applied sciences from the Katholieke Universiteit Leuven, Belgium, in June 1996 and May 2000, respectively. He has been a Research Assistant and a Postdoctoral Fellow of the Fund for Scientific Research-Flanders, Belgium, from October 1996 till September 2003. During that period, Geert Leus was affili-

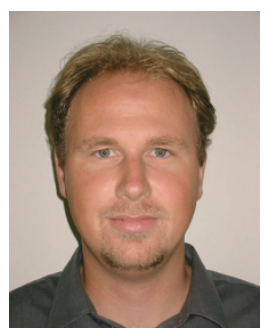
ated with the Electrical Engineering Department of the Katholieke Universiteit Leuven, Belgium. Currently, Geert Leus is an Assistant Professor at the Faculty of Electrical Engineering, Mathematics and Computer Science of the Delft University of Technology, The Netherlands. During the summer of 1998, he visited Stanford University, and from March 2001 till May 2002, he was a Visiting Researcher and Lecturer at the University of Minnesota. His research interests are in the area of signal processing for communications. Geert Leus received a 2002 IEEE Signal Processing Society Young Author Best Paper Award. He is a Member of the IEEE Signal Processing for Communications Technical Committee, and an Associate Editor for the IEEE Transactions on Wireless Communications and the IEEE Signal Processing Letters.

Marc Moonen received the Electrical Engineering degree and the Ph.D. degree in applied sciences from the Katholieke Universiteit Leuven, Leuven, Belgium, in 1986 and 1990, respectively. Since 2004, he is a Full Professor at the Electrical Engineering Department of Katholieke Universiteit Leuven, where he is currently heading a research team of $16 \mathrm{Ph} . \mathrm{D}$. candidates and postdocs, working in the area of signal processing for digital communications, wireless communications, DSL, and audio signal processing. He received the 1994 KU Leuven Research Council Award, the 1997 Alcatel Bell (Belgium) Award (with Piet Vandaele), and was a 1997 "Laureate of the Belgium Royal Academy of Science." He was the Chairman of the IEEE Benelux Signal Processing Chapter (1998-2002), and is currently a EURASIP AdCom Member (European Association for Signal, Speech and Image Processing, from 2000 till now). He is Editor-in-Chief for the "EURASIP Journal on Applied Signal Processing" (from 2003 till now), and a Member of the Editorial Board of "Integration, the VLSI Journal," "IEEE Transactions on Circuits and Systems II" (2002-2003), "EURASIP Journal on Wireless Communications and Networking," and "IEEE Signal Processing Magazine.”
Hugo De Man is Professor of electrical engineering at the Katholieke Universiteit Leuven, Belgium since 1976. He was Visiting Associate Professor at UC Berkeley in 1975 teaching semiconductor physics and VLSI design. His early research was devoted to the development of mixed-signal, switched capacitor, and DSP simulation tools as well as new topologies for high-speed CMOS circuits which lead to the invention of NORA

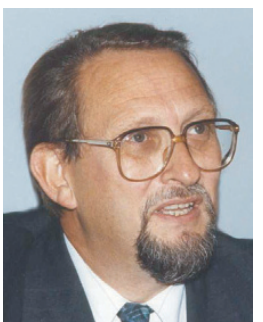
CMOS. In 1984, he was one of the cofounders of IMEC (Interuniversity Microelectronics Center), which, today, is the largest independent semiconductor research institute in Europe with over 1100 employees. From 1984 to 1995, he was Vice President of IMEC, responsible for research in design technology for DSP and telecom applications. In 1995, he became a Senior Research Fellow of IMEC, working on strategies for education and research on design of future post-PC systems. His research at IMEC has lead to many novel tools and methods in the area of high-level synthesis, hardware-software codesign, and $\mathrm{C}++$ based design. Many of these tools are now commercialized by spin-off companies like Coware, Adelante Techn, and Target Compilers. His work and teaching also resulted in a cluster of DSP-oriented companies in Leuven, now known as DSP Valley, where more than 1500 DSP engineers work on design tools and on telecom, networking, and multimedia integrated system products. In 1999, he received the Technical Achievement Award of the IEEE Signal Processing Society, the Phil Kaufman Award of the EDA Consortium, and the Golden Jubilee Medal of the IEEE Circuits and Systems Society. Hugo De Man is an IEEE Fellow and a Member of the Royal Academy of Sciences in Belgium. 